\title{
Star Formation in the Local Universe from the CALIFA Sample. II. Activation and Quenching Mechanisms in Bulges, Bars, and Disks
}

\author{
C. Catalán-Torrecilla ${ }^{1}$ (D) A. Gil de Paz ${ }^{1}$ (1) , A. Castillo-Morales ${ }^{1}$ (D), J. Méndez-Abreu ${ }^{2}$ (D) , J. Falcón-Barroso ${ }^{3,4}$, S. Bekeraite ${ }^{5}$, \\ L. Costantin ${ }^{6}$, A. de Lorenzo-Cáceres ${ }^{2}$, E. Florido ${ }^{7,8}$ (10), R. García-Benito ${ }^{9}$ (i), B. Husemann ${ }^{10}$ (1) , J. Iglesias-Páramo ${ }^{9}$ (i),

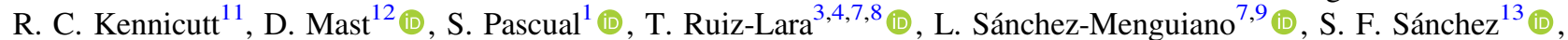 \\ C. J. Walcher ${ }^{5}$ (1) J. Bland-Hawthorn ${ }^{14}$, S. Duarte Puertas ${ }^{9}$, R. A. Marino ${ }^{15}$, J. Masegosa ${ }^{9}$, and P. Sánchez-Blázquez ${ }^{16,17}$
} CALIFA Collaboration

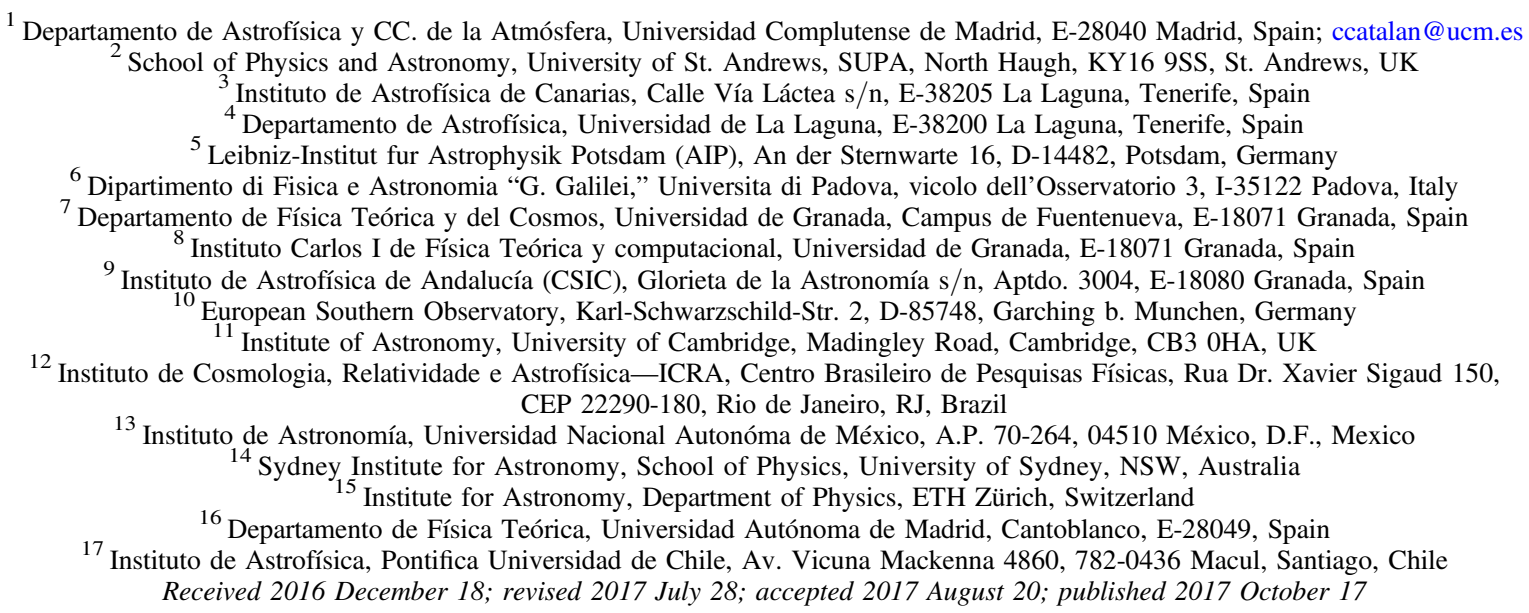

\begin{abstract}
We estimate the current extinction-corrected $\mathrm{H} \alpha$ star formation rate (SFR) of the different morphological components that shape galaxies (bulges, bars, and disks). We use a multicomponent photometric decomposition based on Sloan Digital Sky Survey imaging to Calar Alto Legacy Integral Field Area Integral Field Spectroscopy (IFS) datacubes for a sample of 219 galaxies. This analysis reveals an enhancement of the central SFR and specific $\mathrm{SFR}\left(\mathrm{sSFR}=\mathrm{SFR} / M_{\star}\right)$ in barred galaxies. Along the main sequence, we find that more massive galaxies in total have undergone efficient suppression (quenching) of their star formation, in agreement with many studies. We discover that more massive disks have had their star formation quenched as well. We evaluate which mechanisms might be responsible for this quenching process. The presence of type 2 AGNs plays a role at damping the sSFR in bulges and less efficiently in disks. Also, the decrease in the sSFR of the disk component becomes more noticeable for stellar masses around $10^{10.5} M_{\odot}$; for bulges, it is already present at $\sim 10^{9.5} M_{\odot}$. The analysis of the line-of-sight stellar velocity dispersions $(\sigma)$ for the bulge component and of the corresponding Faber-Jackson relation shows that AGNs tend to have slightly higher $\sigma$ values than star-forming galaxies for the same mass. Finally, the impact of environment is evaluated by means of the projected galaxy density, $\Sigma_{5}$. We find that the SFR of both bulges and disks decreases in intermediate- to high-density environments. This work reflects the potential of combining IFS data with 2D multicomponent decompositions to shed light on the processes that regulate the SFR.
\end{abstract}

Key words: galaxies: evolution - galaxies: spiral - galaxies: star formation - galaxies: structure - techniques: photometric - techniques: spectroscopic

Supporting material: figure set, machine-readable table

\section{Introduction}

Among the multiple open issues on galaxy formation and evolution, arguably the most fundamental are related to the evolution of the baryonic component and, more specifically, the relative role of the different mechanisms that can trigger and quench star formation in galaxies.

For the processes that can activate and regulate star formation, these may vary depending on the location within the galaxy. Secular internal evolution (Kormendy \& Kennicutt 2004) and the accretion of gas (Dekel et al. 2009; Sánchez Almeida et al. 2014) are likely dominant in galaxy disks, with the latter process being progressively more important as we move outward in the disks. In the case of the central regions, in situ star formation is strongly affected by the amount of gas inflow that is driven to the center owing to the presence of bars (Sakamoto et al. 1999; Sheth et al. 2005) or by galaxy mergers (Barnes \& Hernquist 1991).

With respect to the quenching of in situ star formation in galaxies, these are also expected to differ depending on whether we are talking about the formation of stars associated with bulges, bars, or disks. Some of the mechanisms that have been proposed to be responsible for the star formation shutdown are related to the gas consumption, such as the 
termination of gas supply, i.e., strangulation (Kawata \& Mulchaey 2008; Peng et al. 2015), or ram pressure stripping (Book \& Benson 2010; Steinhauser et al. 2016). The previous mechanisms that tranform galaxies are related to the influence of the environment in regulating the star formation rate (SFR) in galaxies (Hashimoto et al. 1998; Koyama et al. 2013). Galaxy harassment (Moore et al. 1996, 1998; Bialas et al. 2015) and morphological quenching (Martig et al. 2009) are also important.

The role of active galactic nuclei (AGNs) in enhancing (Silk 2005, 2013) or suppressing the star formation in the host galaxy (Oppenheimer et al. 2010; Page et al. 2012; Shimizu et al. 2015; Carniani et al. 2016; Hopkins et al. 2016), the effect of SN-driven winds (Bower et al. 2012; Stringer et al. 2012), and the feedback from massive stars (Dalla Vecchia \& Schaye 2008; Hopkins et al. 2012) have important implications for the evolution of galaxies as well.

Different mechanisms act on different spatial scales and are sensitive to the presence of specific structural components (spiral arms, bars, etc.). That is why having high spatial resolution is crucial for solving the problem. Besides, it is also important to quantify how these mechanisms compete not only as a function of different galaxy properties but also as a function of redshift. One of the most fundamental parameters that characterizes galaxies is its SFR. A better understanding of the distribution of the SFR in the different stellar structures that shaped galaxies in the local universe will shed some light on their formation and evolution processes. The advance of Integral Field Spectroscopy (IFS) techniques gives us the opportunity to accurately measure the SFR at the different components that are forming the galaxies, such as unresolved nuclear sources, bulges, bars, and disks. We can also explore the capacity of forming new stars with respect to the stellar mass in each of these stellar structures. This is a determining path if we want to know the different contributions of each component to the integrated value of the SFR in each galaxy. The Calar Alto Legacy Integral Field Area (CALIFA) survey (Sánchez et al. 2012) provides us with excellent data to answer these questions in a spatially resolved way. Some early attempts based on radial profiles of the SFR as a function of galaxy morphology suggest that galaxies are quenched inside out, and that this process is faster in the central, bulge-dominated part than in the disks (González Delgado et al. 2016). Here we perform a more precise analysis by isolating the galaxies in their basic stellar structures. We combine for the first time in a large sample of galaxies the 2D photometric decomposition of the CALIFA galaxies (MendezAbreu et al. 2017) with IFS data to measure the SFR in the different morphological components of galaxies.

This paper is organized as follows: In Section 2, we describe the CALIFA reference sample used in this article. In Section 3, we describe the analysis and methodology applied to the data, including the concept of "smooth aperture"; the 2D photometric decomposition in bulges, bars, and disks; and the derivation of the corresponding IFS-based SFRs. Our results are discussed in Section 4. Finally, in Section 5, we summarize the main conclusions of this work. Throughout our paper we use a cosmology defined by $H_{0}=70 \mathrm{~km} \mathrm{~s}^{-1} \mathrm{Mpc}^{-1}, \Omega_{\Lambda}=0.7$, and a flat universe.

\section{CALIFA Sample}

The galaxies used in this work are part of the CALIFA survey (Sánchez et al. 2012). Data were obtained with the
Potsdam Multi-Aperture Spectrophotometer (Roth et al. 2005) in the PPak mode (Kelz et al. 2006) mounted on the $3.5 \mathrm{~m}$ telescope at the Calar Alto Observatory. As a brief summary, galaxies have spectroscopic redshifts in the range $0.005<$ $z<0.03$ and angular isophotal diameter in the range $45^{\prime \prime}<\mathrm{D} 25<80^{\prime \prime}$ in the Sloan Digital Sky Survey (SDSS) $r$ band. The properties of the CALIFA mother sample are fully described in Walcher et al. (2014).

The observations span the whole optical wavelength range in two overlapping setups. The V500 grating covers the range $3745-7500 \mathrm{~A}$ at a spectral resolution of $R \sim 850$, while the V1200 grating is restricted to $3650-4840 \AA$ but with a higher resolution $(R \sim 1650)$. As our aim is to calculate extinctioncorrected $\mathrm{H} \alpha$ luminosities in each stellar galaxy component, it is desirable to have both $\mathrm{H} \beta$ and $\mathrm{H} \alpha$ emission lines in the same observing range. This is the reason why we use the V500 setup throughout this work. The V1200 data are restricted to the analysis of the line-of-sight (LOS) velocity dispersions (Section 4.4.1).

This paper makes use of 545 CALIFA galaxies that have been observed and processed with the V500 grating, are part of the Data Release 3 (DR3; Sánchez et al. 2016), and belong to the CALIFA mother sample. This criterion should guarantee that we maintain the limits where the mother sample is representative of the general galaxy population: 9.7 and 11.4 in $\log \left(M_{\star} / M_{\odot}\right),-19.0$ and -23.1 in $r$-band absolute magnitude, and 1.7 and $11.5 \mathrm{kpc}$ in half-light radius (Walcher et al. 2014). As we are interested in the SFR properties of these galaxies in their different components, our sample is further constrained to those galaxies that are eligible for the 2D photometric decomposition. Galaxies meeting any of the following criteria were excluded: (1) if they are forming a pair, are forming an interacting system, or have a heavily distorted morphology; and (2) if they are highly inclined galaxies, as the projection effects can affect the results (typically $i>70^{\circ}$ ). More details about the sample selection are given in Mendez-Abreu et al. (2017). A total of 204 galaxies were excluded in this way. We also reject 122 galaxies that do not show detectable $\mathrm{H} \alpha$ emission based on a signal-to-noise ratio $(\mathrm{S} / \mathrm{N})$ criterion including also galaxies classified as elliptical in the $2 \mathrm{D}$ decomposition analysis. We impose a minimum of $\mathrm{S} / \mathrm{N}>5$ for the detection of both $\mathrm{H} \beta$ and $\mathrm{H} \alpha$ emission lines in each photometric structure of the galaxies (more details are given in Section 3.3). This leads to the final sample of 219 CALIFA galaxies for this work.

\section{Analysis}

In this section we describe the method applied to obtain an extinction-corrected $\mathrm{H} \alpha$ SFR value for each galaxy morphological component (nuclear point source, bulge, bar, and disk). This method relies on the combination of 2D decomposition of multiband photometry on IFU spectral datacubes. Consequently, our galaxy components are defined based exclusively on the fitting to the photometry. Our objective is to determine how these components will grow in stellar mass owing to in situ star formation, which ultimately dominates the total mass growth in the local universe. We aim to identify the mechanism(s) that either trigger or quench star formation in each of these regions, and therefore in galaxies as a whole, but going beyond the use of simple ill-defined spectrophotometric apertures on the datacubes. 


\subsection{Assigning SFR Values to Morphological Components Defined Based on Purely Photometric Criteria}

Different approaches can be used to perform a spatially resolved analysis of the SFR in galaxies, including individual pixels, full 2D maps, radial profiles, or, as in this paper, multicomponent decomposition. The analyses based on 2D maps or individual pixels have difficulty in combining information from different galaxies and are also limited in our case by the coarse spatial resolution of the CALIFA datacubes. A simplified approach would have been to identify the transition radius between the bulge and disk components in 1D surface brightness profiles and use this radius (and the galaxy ellipticity and position angle (PA) at that radius) to define spectrophotometric apertures for those two components. However, early tests already showed that this 1D approach allows us neither to properly isolate the emission coming from the bulge and the disk nor to deal with objects where a clear bar is present. In fact, some studies (Aguerri et al. 2005; Weinzirl et al. 2009; Meert et al. 2015) have demonstrated the importance of including the bar to obtain the precise parameters for the bulge component.

An alternative would have been to use the distinct kinematic features of bulge, disk, and bar main-sequence stars to separate components. However, it is quite likely that the stars currently being formed do not follow the same balance but might all form in a kinematically cold component and are being heated up afterward. In addition, for this particular aim, the CALIFA spectral resolution is at the limit of what is needed to perform such multicomponent kinematical fitting. Future highefficiency IFS facilities working at $R>5000$, such as MEGARA (at GTC; Gil de Paz et al. 2016) or WEAVE (at WHT; Dalton et al. 2014), will help in that regard.

To overcome the previous limitations, we introduce here the concept of "smooth aperture" as the optimal option in our case. Instead of imposing a fixed aperture to define and isolate the different galaxy components (which would have to be defined based on terms of corresponding scale lengths), we allow the light associated with each spaxel in the datacube to have a contribution coming from different components (bulge, bar, disk). The starting stellar structure parameters are recovered using a multicomponent decomposition, as it has been widely proved to be one of the best methods for that purpose (de Souza et al. 2004; Gadotti 2009; Weinzirl et al. 2009; Meert et al. 2015, 2016; Salo et al. 2015), and these parameters are obtained based exclusively on photometric criteria (see Section 3.2).

Bulge and disk SFR values are assigned as those measured in regions where the stellar content is dominated by stars that follow either a bulge or disk light profile. In particular, the central regions of galaxies could be classified as either classical bulges or pseudobulges. The latter ones are thought to have a complex star formation history where young stellar populations could be present. Nevertheless, we do not aim to probe the mechanism by which the stars were formed in each component, but to provide a measurement of its current SFR focusing on the stars that are associated with their spatial distribution at present. Consequently, these are also the regions where the SFR is expected to later contribute to the growth of the stellar mass of these components.

\subsection{D Photometric Decomposition Analysis}

We use the structural parameters derived for the CALIFA galaxies in Mendez-Abreu et al. (2017). These values were obtained by applying the 2D photometric decomposition code GASP2D (Méndez-Abreu et al. 2008, 2014) over the $g$-, $r$-, and $i$-band images from the Sloan Digital Sky Survey Data Release 7 (SDSS-DR7, Abazajian et al. 2009). The use of SDSS images is justified in terms of their higher spatial resolution in comparison with CALIFA making the method more precise. GASP2D makes use of the widely used Levenberg-Marquardt algorithm (i.e., damped least-squares method) to fit the 2D surface brightness distributions of galaxies. This code allows the simultaneous fitting of different galaxy structures such as nuclear point sources, bulges, bars, and disks (including breaks). The reader is referred to Mendez-Abreu et al. (2017) for more details about the methodology of the fitting algorithm.

For the purpose of this work, we use the parameters derived using the SDSS $g$-band images. This band is the one that provides the best compromise between image depth and being able to fit analytic functions to the light distribution of the youngest possible stellar populations. We have discarded the use of other bands for the following reasons: (a) trying to fit these 2D analytic components to the UV bands leads to catastrophic failures in all but the very early type systems, (b) the $u$ band is significantly less deep than $g$ in SDSS (and it is subject to the same problems as the UV, especially in latetype spirals), and (c) redder bands would progressively trace older stellar populations. It is worth emphasizing here that the main objective of the use of the $g$-band data is to provide relative weights for the different components in those regions where they compete in surface brightness (inner disk, outer regions of the bar, etc.). However, the actual SFR is dominated by the amount of extinction-corrected $\mathrm{H} \alpha$ luminosity provided by the CALIFA datacubes to which these weights are applied.

The process followed to create the datacubes for each of the stellar components in each galaxy is the following. First, we create the photometric characterization of the multiple stellar structures (nuclear point source, bulge, bar, or disk), i.e., their best-fitting 2D g-band models as illustrated in the left panels of Figure 1. Then, we create weight maps for each stellar component. The weight maps are defined as the ratio between the light in each galaxy structure (nuclear point source, bulge, bar, or disk) and the total luminosity of the galaxy, as given by the SDSS $g$-band best-fitting models. These weight maps are computed for each individual CALIFA spaxel. Finally, the original CALIFA datacube of the galaxy is multiplied by these weight maps. This means that a $3 \mathrm{D}$ datacube is now created for each of the photometric structures.

Figure 1 illustrates the process. The complete figure set (219 images) is available in the online version of the journal. Once we have created the final weighted datacube for each component, we can obtain the corresponding distribution of the continuum-subtracted $\mathrm{H} \alpha$ luminosity. Middle panels of Figure 1 show the continuum-subtracted $\mathrm{H} \alpha$ luminosity for the disk, the bar, and the bulge (from top to bottom). We emphasize that these $\mathrm{H} \alpha$ maps are given as a visual tool to prove the goodness of the method but that the actual $\mathrm{H} \alpha$ luminosity is computed using the corresponding spectrum per component as explained in the next paragraph. We have identified 15 galaxies (7\% of the bulge components) in the online figure set that show a clear contamination coming from 
Relative Flux (g-band)
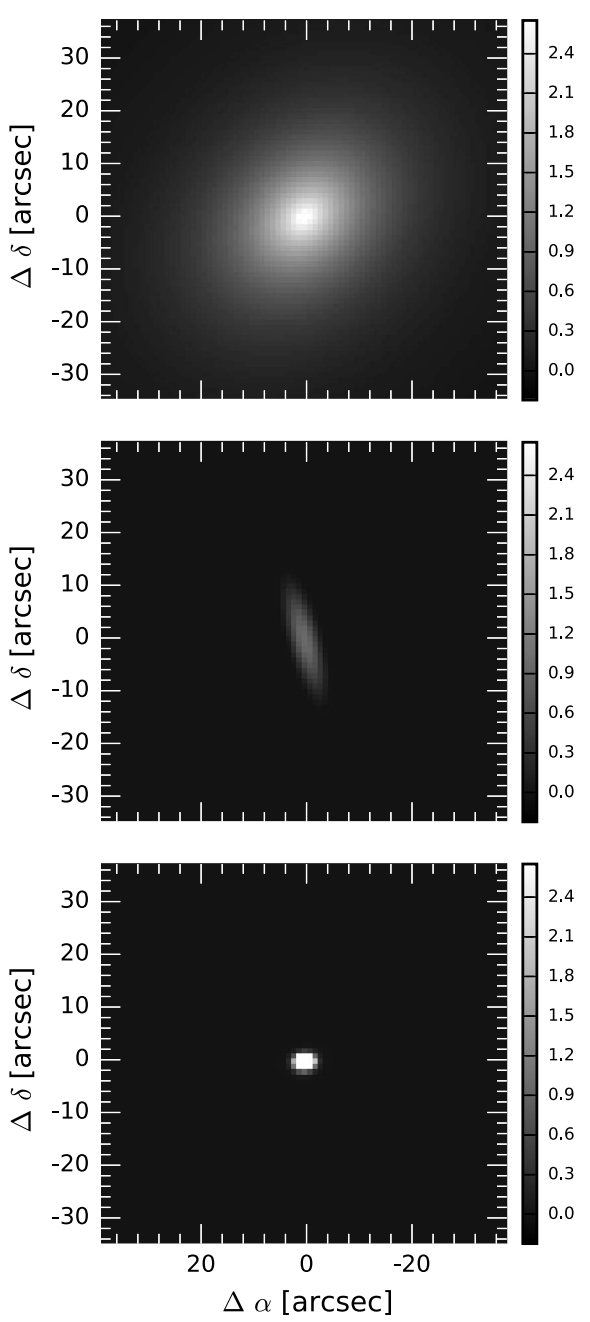

Flux $(\mathrm{H} \alpha)$

$\left[10^{-16} \mathrm{erg} \mathrm{s}^{-1} \mathrm{~cm}^{-2}\right]$
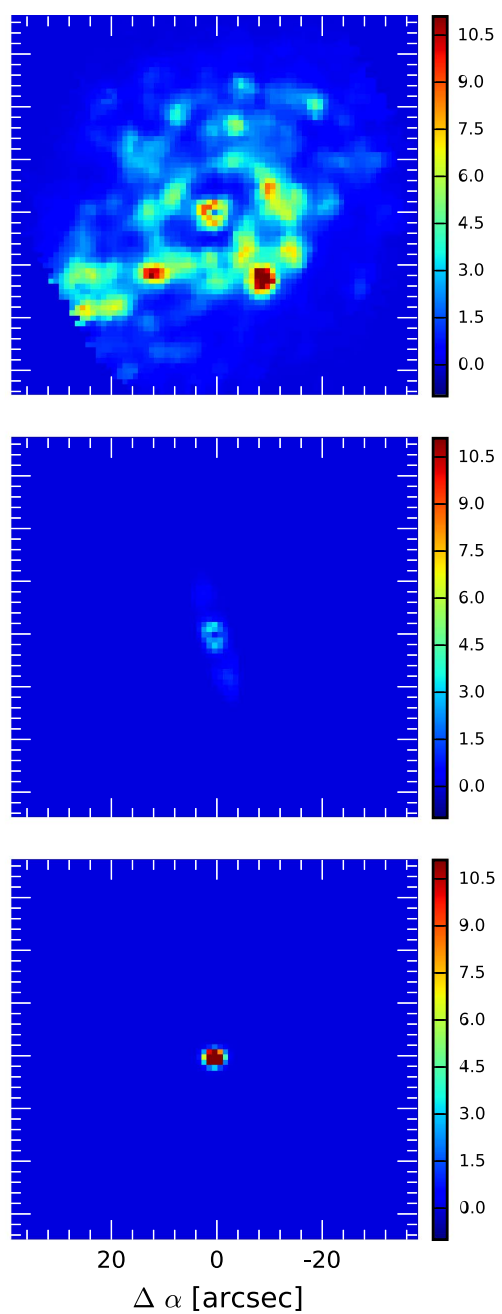

NGC 2253
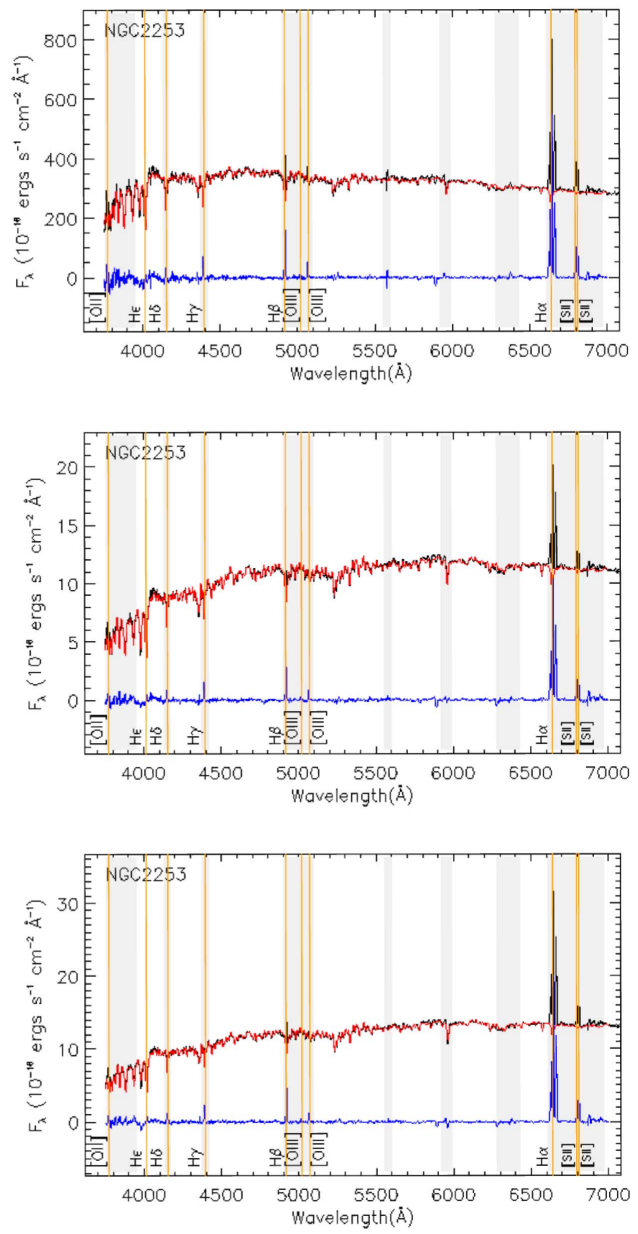

Figure 1. Left panels: 2D g-band models derived from the multicomponent photometric decomposition. Disk, bar, and bulge components are shown from top to bottom, respectively. Units for the flux are given relative to the central surface brightness of the bar component (if the bar is not present, the central surface brightness of the disk component is used instead). Central surface brightness and the rest of the parameters needed to create these $g$-band models are provided in Mendez-Abreu et al. (2017). Middle panels: distribution of the continuum-subtracted $\mathrm{H} \alpha$ luminosity in the different stellar galaxy components. To create these $\mathrm{H} \alpha$ maps, the original CALIFA datacubes have been multiplied by the corresponding weight maps in each morphological component (i.e., there is a weighted datacube for each galaxy component) and then analyzed spaxel by spaxel. Right panels: integrated spectrum extracted for the weighted datacube for each galaxy structure (bulge, bar, and disk) is shown in black. Gray-colored vertical ranges correspond to the emission lines and sky lines masked out during the fitting procedure. The red spectrum corresponds to the best fit for the underlying stellar population. The emission-line spectrum originated by the ionized gas is shown in blue. The latest is the one used to measure the $\mathrm{H} \alpha$ and $\mathrm{H} \beta$ fluxes associated with each component. The complete figure set (219 images) showing the models, $\mathrm{H} \alpha$ maps, and their corresponding spectra for each of the galaxies used in this work is available in the electronic edition of the journal.

(The complete figure set (219 images) is available.)

the internal parts of the disks. These objects are marked with a nuclear $3^{\prime \prime}$ green aperture (see Section 3.5).

Finally, we obtain the integrated spectrum for each galaxy structure (right panels of Figure 1) and the corresponding $\mathrm{H} \alpha$ flux to derive the SFR. The analysis of the spectra extracted from the CALIFA datacubes is explained in detail in Section 3.3, and it is similar to that described in CatalánTorrecilla et al. (2015). We emphasize here that the spectra obtained for each component might not be optimal for the study of intermediate-age to old stellar populations in each of these regions since these (more evolved) populations do show distinct kinematical properties in bulges, bars, and disks that could be used instead (Johnston et al. 2017; Tabor et al. 2017).
Indeed, these properties are the ones that ultimately define what bulges, bars, and disks truly are.

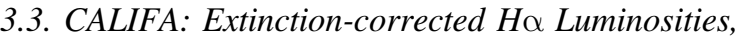 Continuum Subtraction, and Line-flux Measurements}

Once we have the final datacube for each component, we obtain the integrated spectra (see below) and the corresponding $\mathrm{H} \alpha$ fluxes.

For each component (nuclear point source, bulge, bar, and disk), we spatially integrate their corresponding datacube to generate an integrated spectrum using an elliptical aperture with a major-axis radius of $36^{\prime \prime}$. The use of $36^{\prime \prime}$ apertures is justified in terms of assuring a homogenous way for computing 
the aperture effects that are mentioned at the end of this section. The minor-to-major-axis ratio of the elliptical aperture is given by the isophotal major and minor axis in the $g$ band from SDSS-DR7, as well as the isophotal PA. Before extracting the integrated spectra, a spatial masking over the datacubes is performed to avoid light coming from spaxels contaminated by field stars or background objects.

The complete description of the methodology applied to obtain the $\mathrm{H} \alpha$ and $\mathrm{H} \beta$ fluxes is explained in detail in CatalánTorrecilla et al. (2015). For the sake of completeness, we briefly describe here the main steps. Once we have the integrated spectrum of each component, we carefully remove the stellar continuum using a linear combination of two single stellar population (SSP) evolutionary synthesis models from Vazdekis et al. (2010) based on the MILES stellar library (Sánchez-Blázquez et al. 2006). Two sets of models with a Kroupa initial mass function (IMF; Kroupa 2001) are combined. One set contains models (considered as a young stellar population) with ages of $0.10,0.50$, and $0.79 \mathrm{Gyr}$. A second set (considered as an old stellar population) involves ages of 2.00, 6.31, and 14.13 Gyr. For each age we considered five different metallicities with $[\mathrm{M} / \mathrm{H}]$ values equal to 0.00 , $0.20,-0.40,-0.71$, and -1.31 dex offset from the solar value. The basic steps applied to obtain the $\mathrm{H} \alpha$ and $\mathrm{H} \beta$ fluxes are the following: (1) to shift the SSP templates to match the systemic velocity of the integrated spectrum; (2) to convolve each stellar population model with a Gaussian profile so the absorption features could be broadened to match those of the integrated spectrum; (3) to redden the spectrum using a $k(\lambda)=R_{V}$ $(\lambda / 5500 \AA)^{-0.7}$ power law, where $R_{V}=5.9$, as given by Charlot \& Fall (2000); and (4) to determine the best linear combination of SSPs by a $\chi^{2}$ minimization. Finally, $\mathrm{H} \alpha$ and $\mathrm{H} \beta$ fluxes are obtained from fitting Gaussians to the pure emission-line spectra. The flux uncertainties are estimated from a random redistribution of the residuals after the Gaussian fittings mentioned before. The procedure, which consists of adding this new residual spectrum to the pure emission-line spectrum and performing afterward the Gaussian fittings, is repeated 1000 times. The standard deviation of the computed fluxes is taken as the error in the $\mathrm{H} \alpha$ and $\mathrm{H} \beta$ fluxes.

An important parameter to take into account is the amount of dust attenuation for our measured $\mathrm{H} \alpha$ luminosities. In particular, we use Balmer decrements with a Galactic extinction curve and a foreground screen dust geometry approximation to estimate the attenuation. Although there are not a considerable number of edge-on galaxies in this work owing to the selection criteria imposed for the $2 \mathrm{D}$ photometric decomposition, we refer the reader to the extensive analysis in Catalán-Torrecilla et al. (2015), where we test that the use of the Balmer decrement in the foreground dust screen approximation does not have an important impact on the SFR derived for these galaxies.

As some galaxies could extend beyond the PPak field of view (FOV), we have applied aperture corrections to our extinction-corrected $\mathrm{H} \alpha$ measurements. Among all the morphological components analyzed, the light coming from the disk is the only one that might extend beyond the FOV. As a consequence, we have applied these aperture corrections to the spectrum of the disk only. We have derived dust-corrected $\mathrm{H} \alpha$ growth curves using elliptical integrated apertures centered at the center of mass of the galaxy, with radii increasing by steps of $3^{\prime \prime}$ up to a maximum radius of $36^{\prime \prime}$ (a similar methodology is used in Gil de Paz et al. 2007). The last aperture corresponds to the $36^{\prime \prime}$ aperture that was used previously to compute the integrate disk spectra. This method allows us to estimate the aperture effects in all the disks that create our sample in a uniform way. Then, we calculate the gradients of the extinction-corrected $\mathrm{H} \alpha$ growth curves as the ratio between the flux in each aperture and the corresponding radial interval. This gradient decreases and becomes nearly zero when it approaches the maximum radius, as the flux tends to be constant in the last apertures. Finally, if we plot the flux as a function of these gradients, the intercept of this relation gives us the value of the aperture correction. The mean and the median values for the aperture correction multiplicative factors in our sample are 1.19 and 1.08, respectively. The extinctioncorrected $\mathrm{H} \alpha \mathrm{SFR}$ measurements for each galaxy component are given in Table 1.

\subsection{CALIFA: Stellar Masses}

Stellar mass is a key parameter on the process of formation and evolution of galaxies. For this study, we rely on the CALIFA total stellar masses that were calculated by Walcher et al. (2014) using Bruzual \& Charlot (2003) stellar population models with a Chabrier (2003) stellar IMF to construct UV to NIR SEDs. In particular, far-UV (FUV) (GALEX; Martin et al. 2005), $u, g, r, i, z$ (SDSS-DR7; Abazajian et al. 2009), and $J, H, K$ (2MASS Extended Source Catalog; Jarrett et al. 2000) photometric data were used.

We are interested in determining the stellar masses not only for the galaxies as a whole but also for their different structural components. For that reason, we apply the recipe below that allows deriving the mass in each component using the $i$-band mass-to-light relation of each component, $\left(M_{*} / L\right)_{\text {comp }, i}$, the galaxy total stellar mass, $M_{* \text {,total }}$, and the bulge-to-total $(\mathrm{B} / \mathrm{T})$, bar-to-total (Bar/T), and disk-to-total (D/T) luminosity ratios in the $i$ band. The luminosity ratios are derived as by-products of the 2D photometric decomposition for our galaxies in $i$ and $g$ bands (see Section 3.2 for more details). We use the $i$-band values, as they will better reproduce the stellar mass distribution than the $g$ band. Thus, we obtain

$$
\frac{M_{*, \mathrm{comp}}}{M_{*, \text { total }}}=\frac{\left(\frac{M_{*}}{L}\right)_{\mathrm{comp}, i}}{\left(\frac{M_{*}}{L}\right)_{\mathrm{total}, i}} \cdot \frac{L_{\mathrm{comp}, i}}{L_{\mathrm{total}, i}} .
$$

We make use of the color-dependent $M_{*} / L_{i}$ ratio given by Equation (7) in Taylor et al. (2011), where the authors also assume a Chabrier (2003) IMF. The authors proposed the following empirical relation between $M_{*} / L_{i}$ and $(g-i)$ color:

$$
\log M_{*} / L_{i}=-0.68+0.70(g-i) \text {. }
$$

In our case, the $(g-i)$ colors correspond to $(g-i)_{\text {disk }}$,

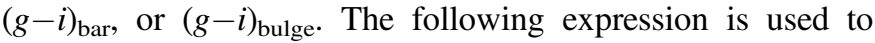
obtain the $(g-i)$ colors for each galaxy component:

$$
(g-i)_{\mathrm{comp}}=(g-i)_{\text {total }}-2.5 \cdot \log \frac{\left(\frac{L_{\text {comp }}}{L_{\text {total }}}\right)_{g}}{\left(\frac{L_{\text {comp }}}{L_{\text {total }}}\right)_{i}} .
$$

The $(g-i)_{\text {total }}$ color measurements came from the analysis of the growth curve magnitudes performed in Walcher et al. (2014).

To verify the goodness of our stellar mass values per component, we have checked that the sum of the stellar masses for the different components obtained via the previous 
Table 1

Galaxy Sample and Measurements of SFR by Galaxy Components

\begin{tabular}{|c|c|c|c|c|c|c|c|c|c|c|c|}
\hline$\overline{\mathrm{ID}}$ & $\begin{array}{l}\text { Name } \\
\text { (2) }\end{array}$ & $\begin{array}{c}M_{\mathrm{BULGE}} \\
\left(\times 10^{10} M_{\odot}\right) \\
(3)\end{array}$ & $\begin{array}{c}M_{\mathrm{BAR}} \\
\left(\times 10^{10} M_{\odot}\right) \\
(4)\end{array}$ & $\begin{array}{c}M_{\text {DISK }} \\
\left(\times 10^{10} M_{\odot}\right) \\
(5)\end{array}$ & $\begin{array}{c}\mathrm{SFR}\left(\mathrm{H} \alpha_{\mathrm{BULGE}}\right) \\
\left(M_{\odot} \mathrm{yr}^{-1}\right) \\
(6)\end{array}$ & $\begin{array}{c}\mathrm{SFR}\left(\mathrm{H} \alpha_{\mathrm{BAR}}\right) \\
\left(M_{\odot} \mathrm{yr}^{-1}\right) \\
(7)\end{array}$ & $\begin{array}{c}\mathrm{SFR}\left(\mathrm{H} \alpha_{\text {DISK }}\right) \\
\left(M_{\odot} \mathrm{yr}^{-1}\right) \\
(8)\end{array}$ & $\begin{array}{l}\text { HT } \\
(9)\end{array}$ & $\begin{array}{c}\Sigma_{5} \\
\left(\mathrm{Mpc}^{-2}\right) \\
(10)\end{array}$ & $\begin{array}{l}\text { AGN } \\
\text { (11) }\end{array}$ & $\begin{array}{c}\sigma_{\text {bulge }} \\
\left(\mathrm{km} \mathrm{s}^{-1}\right) \\
(12)\end{array}$ \\
\hline 2 & UGC 00005 & $0.30 \pm 0.02$ & $\ldots$ & $5.54 \pm 0.30$ & $0.040 \pm 0.003$ & $\ldots$ & $3.890 \pm 0.155$ & $\mathrm{Sbc}$ & $\ldots$ & yes & $106.24 \pm 1.98$ \\
\hline 3 & NGC 7819 & $0.16 \pm 0.03$ & $0.22 \pm 0.04$ & $0.85 \pm 0.17$ & $0.605 \pm 0.008$ & $0.268 \pm 0.009$ & $0.609 \pm 0.021$ & $\mathrm{Sc}$ & $\ldots$ & no & $68.29 \pm 1.55$ \\
\hline 5 & IC 1528 & $0.20 \pm 0.00$ & $\ldots$ & $1.07 \pm 0.01$ & $0.031 \pm 0.001$ & $\ldots$ & $1.017 \pm 0.023$ & $\mathrm{Sbc}$ & $\ldots$ & no & $55.40 \pm 1.60$ \\
\hline 6 & NGC 7824 & $5.41 \pm 0.12$ & $\ldots$ & $8.78 \pm 0.20$ & $0.060 \pm 0.009$ & $\ldots$ & $0.201 \pm 0.017$ & $\mathrm{Sab}$ & $\ldots$ & no & $210.19 \pm 4.03$ \\
\hline 7 & UGC 00036 & $1.26 \pm 0.04$ & $1.14 \pm 0.03$ & $4.89 \pm 0.15$ & $0.051 \pm 0.003$ & $0.087 \pm 0.006$ & $0.530 \pm 0.052$ & $\mathrm{Sab}$ & $\ldots$ & no & $173.99 \pm 2.70$ \\
\hline 8 & NGC 0001 & $1.68 \pm 0.20$ & $\ldots$ & $2.70 \pm 0.33$ & $1.586 \pm 0.051$ & $\ldots$ & $1.021 \pm 0.042$ & $\mathrm{Sbc}$ & $\ldots$ & no & $111.93 \pm 1.42$ \\
\hline 10 & NGC 0036 & $0.65 \pm 0.08$ & $0.92 \pm 0.11$ & $5.58 \pm 0.65$ & $0.411 \pm 0.027$ & $0.215 \pm 0.012$ & $1.210 \pm 0.105$ & $\mathrm{Sb}$ & $\ldots$ & yes & $134.06 \pm 2.90$ \\
\hline
\end{tabular}

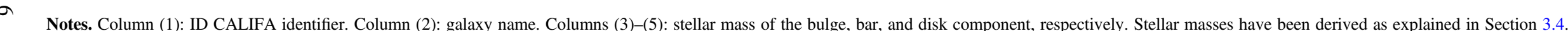

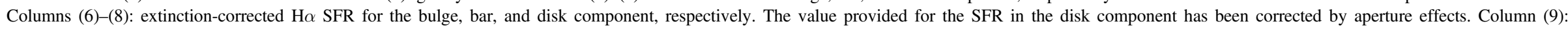

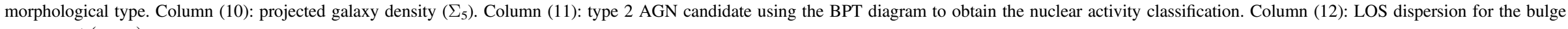
component $\left(\sigma_{\text {bulge }}\right)$

${ }^{\mathrm{a}}$ These galaxies have a nuclear point-source component instead of a bulge component.

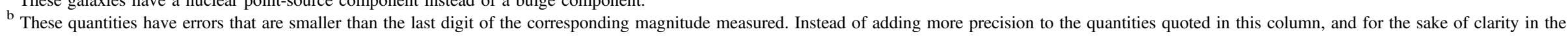
formatting of the table, we have not included their errors here.

(This table is available in its entirety in machine-readable form.) 
equations reproduces the total stellar mass derived from SED fitting for each galaxy. Both methods yield similar results for $82 \%$ of the galaxies, with the difference between the sum of the stellar components and the SED stellar mass being less than $\pm 15 \%$. For the remaining $18 \%$ of the galaxies, a larger difference arises owing to significant variations in the $(\mathrm{B} / \mathrm{T})_{i}(\mathrm{~B} / \mathrm{T})_{g}$ ratio. The latter case has a mean value of 2.26 for the $(\mathrm{B} / \mathrm{T})_{i}(\mathrm{~B} / \mathrm{T})_{g}$ ratio, in contrast to a value of 1.30 for the cases in which the sum of the derived stellar masses of the components and the SED total stellar mass are similar. The former case is a consequence of the nonlinearity between the luminosity ratios in both bands and the mass-luminosity relation in Equation (2).

As a final remark, we note here that the $\mathrm{H} \alpha$ extinctioncorrected SFR tracer used along this work and the stellar population models applied for the continuum subtraction (Section 3.3) are both based on a Kroupa (2001) IMF. For the sake of consistency, we rescale the stellar masses derived in this section to the Kroupa (2001) IMF, applying the factor 1.08 as obtained in Madau \& Dickinson (2014). This value is almost independent of the stellar population age and has a very weak dependence on metallicity. The stellar masses derived for each galaxy component are provided in Table 1.

\subsection{AGN Optical Classification}

AGN feedback is one of the mechanisms proposed to explain the quenching of the star formation in classical bulges and in massive galaxies, as it has been put forward to explain the differences between models and observations mainly at the high end of the galaxy luminosity function (Silk \& Mamon 2012). Therefore, it is critical to determine which of our CALIFA galaxies host an AGN. We apply a classical emission-line diagnostics to classify the objects into starforming or type 2 AGNs. For that purpose, we use the [O III]/ $\mathrm{H} \beta$ versus $[\mathrm{N}$ II] $/ \mathrm{H} \alpha$ diagram introduced by Baldwin et al. (1981), with the demarcation lines of Kauffmann et al. (2003) and Kewley et al. (2001). We extract the spectrum centered within $3^{\prime \prime}$ of the nucleus, and we imposed an $\mathrm{S} / \mathrm{N}>4$ for the previous emission lines. We obtain that 74 out of 219 galaxies are Seyfert/LINER. From now on, we refer to Seyfert/LINER as type 2 AGN objects. We highlight that galaxies that have type-1 AGN signatures are excluded from the sample completely. In the Unified Model, the emission from the AGNs in Seyfert 1 galaxies outshines that due to recently formed stars, as the broad-line region (BLR) is directly observable, while in Seyfert 2 the BLR is highly obscured and the line emission from the AGNs competes with that due to star formation. Alternatively, several studies have pointed out a different scenario where Seyfert 1 and Seyfert 2 might indeed be different classes of objects, suggesting that Seyfert 2 objects intrinsically lack the BLR (Tran 2001; Tran et al. 2011). Since the spatial resolution in our data is not enough to disentangle whether the central contribution is coming totally from the AGN or it has some contamination from (or even dominated by) star formation, for the type 2 AGN objects we have decided to include them in our sample and to distinctly mark them as type 2 AGNs when necessary. Table 1 provides information about the galaxies classified as AGNs in our sample.

Although LINERs have been traditionally associated with low-luminosity active galactic nuclei (LLAGN; Ho et al. 1993; Terashima et al. 2000), some authors have recently claimed the importance of differentiating between galaxies hosting a weakly active nucleus and galaxies that could be ionized by hot, low-mass evolved stars (a recent discussion about the nature of LINER galaxies is provided by Singh et al. 2013). In that regard, Stasińska et al. (2008) and Cid Fernandes et al. $(2010,2011)$ have proposed to use the observed $\mathrm{H} \alpha$ equivalent widths $\left(\mathrm{EW}_{\mathrm{H} \alpha}\right)$ versus the $[\mathrm{N} \mathrm{II}] / \mathrm{H} \alpha$ ratio in the so-called WHAN diagram, in which the division between weak AGNs and galaxies that are ionized by their hot, low-mass evolved stars is fixed at $3 \AA$. We restrict the estimation of the $\mathrm{EW}_{\mathrm{H} \alpha}$ to the center of our galaxies, i.e., the central $3^{\prime \prime}$, instead of using the total integrated spectrum, as we want to know whether or not the AGN is the dominant photoionization mechanism in the nuclear regions. The $3 \AA$ criterion admittedly overestimates the number of galaxies classified as "retired galaxies," as diluted bona fide AGNs could also be included in this category (Cid Fernandes et al. 2011). For that reason, we analyze the trend for the $6 "$ and $9 "$ apertures in these objects. Radial $\mathrm{EW}_{\mathrm{H} \alpha}$ profiles using CALIFA data have been previously probed to be optimal for the study of the nuclear and extranuclear nebular emission of the warm ionized gas (Gomes et al. 2016). We find that there are two distinct types. On one hand, some galaxies show an increase in the $\mathrm{EW}_{\mathrm{H} \alpha}$ and a reduction in the $[\mathrm{N} \mathrm{II}] / \mathrm{H} \alpha$ ratio at larger apertures, reflecting the presence of a star-forming component. Even more, the integrated spectrum shows values of the $\mathrm{EW}_{\mathrm{H} \alpha}$ larger than $3 \AA$. On the other hand, there are galaxies for which the $\mathrm{EW}_{\mathrm{H} \alpha}$ decreases while the $[\mathrm{N} \mathrm{II}] / \mathrm{H} \alpha$ ratio maintains a roughly constant value when using larger apertures. There are two possibilities for this case: (a) the evolved stars that are responsible for the photoionization of these regions exhibit a gradient that might explain the radial variation in $\mathrm{EW}_{\mathrm{H} \alpha}$, and/or (b) there is actually an AGN in the central region and the older populations in their surroundings create a decline in the $\mathrm{EW}_{\mathrm{H} \alpha}$ measurements. Whether one or both of these possibilities is the responsible mechanism is beyond the scope of this paper. There are still, however, a fraction of $39.2 \%$ of the galaxies initially classified as AGNs (33.5\% of the sample), where a homogeneous population of evolved stars could generate, according to the predictions of Cid Fernandes et al. (2011), the $\mathrm{EW}_{\mathrm{H} \alpha}$ values and distribution observed (at least at the spatial resolution of CALIFA). Thus, galaxies that have a Seyfert/LINER central spectrum are referred as type 2 AGNs even though a fraction of these could be actually powered by a source distinct from a truly AGN.

\section{Results}

In this work we use extinction-corrected $\mathrm{H} \alpha\left(\mathrm{H} \alpha_{\text {corr }}\right)$ as our SFR reference indicator following the recipe given by Kennicutt \& Evans (2012). From now on we will use $\mathrm{H} \alpha$ instead of $\mathrm{H} \alpha_{\text {corr }}$ to shorten the term throughout the text, although we emphasize that all the $\mathrm{H} \alpha$ SFR measurements used here are extinction corrected.

We have previously investigated the goodness of $\mathrm{H} \alpha$ as an SFR tracer for a representative sample of 272 CALIFA galaxies (for more details see Catalán-Torrecilla et al. 2015). For that purpose, we compared extinction-corrected $\mathrm{H} \alpha$ integrated measurements with single-band (FUV corr, $22 \mu \mathrm{m}$, and total-IR $(8-1000) \mu \mathrm{m}$ (TIR)) and hybrid $\left(22 \mu \mathrm{m}+\mathrm{H} \alpha_{\text {obs }}\right.$, TIR + $\mathrm{H} \alpha_{\text {obs }}, 22 \mu \mathrm{m}+\mathrm{FUV}_{\text {obs }}$, TIR $+\mathrm{FUV}_{\text {obs }}$ ) tracers. The latter shows an excellent agreement with dispersions around 0.18 dex. We also find that only $1 \%$ of our objects host highly obscured star formation. Bearing in mind the above considerations, we can safely conclude that the use of extinctioncorrected $\mathrm{H} \alpha$ is appropriate for our sample. Whether or not this 


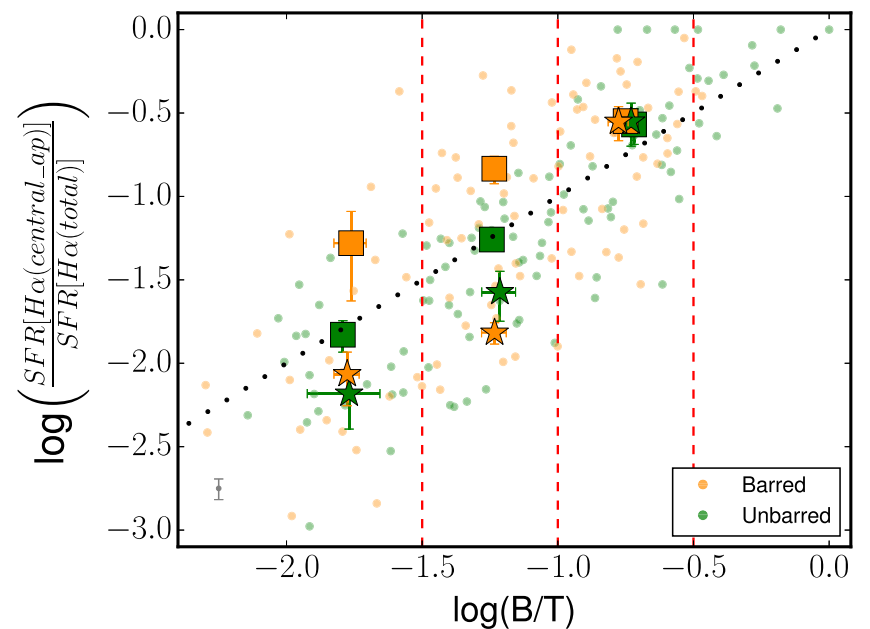

Figure 2. Variation of the $\mathrm{SFR}[\mathrm{H} \alpha$ (central)]/SFR[H $\alpha$ (total)] ratio with the $\mathrm{B} / \mathrm{T}$ parameter obtained for the $g$ band. The central SFR refers to the amount of SFR in the smooth aperture of the bulge component for unbarred (green symbols) and barred galaxies (orange symbols). Squares correspond to purely star-forming galaxies, while stars refer to type 2 AGN objects. The previous symbols correspond to the logarithm of the mean value for the SFR[Ha (central)]/SFR[H $\alpha$ (total)] ratio, and the errors represent the standard deviation of the mean.

calibration can be applied to other samples in the local universe or to higher redshifts depends strongly on the expected fraction of galaxies and SFR that could be locked into completely obscured star-forming sites and also on the percentage of nuclear line emission in Seyfert 2 objects coming from either SF or AGNs (or even ionization from evolved stars).

In this section we show the correlations found between the SFR in the different morphological components of the galaxies and other physical properties such as stellar mass, morphological type, the presence of an AGN, environment, and stellar velocity dispersion. Among other aspects, we investigate the so-called main sequence of galaxies using not only integrated values but also the values in each galaxy morphological component (i.e., nuclear point sources, bulges, bars, and disks).

\subsection{SFR Ratios by Components: SFR Central Enhancement Due to the Presence of Bars}

In this section, we explore the connection between the central $\operatorname{SFR}(\mathrm{H} \alpha)$ and other parameters such as the morphological type and the $\mathrm{B} / \mathrm{T}$ in the $g$ band. Galaxy morphologies were inferred by a combination of independent visual classifications carried out by members of the CALIFA collaboration as described in Walcher et al. (2014), while $\mathrm{B} / \mathrm{T}$ values in the $g$ band came from the analysis of the 2D decomposition (Section 3.2).

The analysis is performed only for galaxies that do show $\mathrm{H} \alpha$ emission in the central regions. For the discussion below, central regions refer to the amount of SFR found in the aperture associated with the bulge component. To investigate whether the impact of the bar could trigger the star formation in the centers of galaxies, galaxies are classified into two main types, barred (orange symbols) and unbarred (green symbols), in Figures 2 and 3, respectively.

The bulges in our sample could be either classical bulges or pseudobulges (for an extensive review see Kormendy \& Kennicutt 2004). Although with limitations, one can broadly discriminate between classical bulges and pseudobulges using

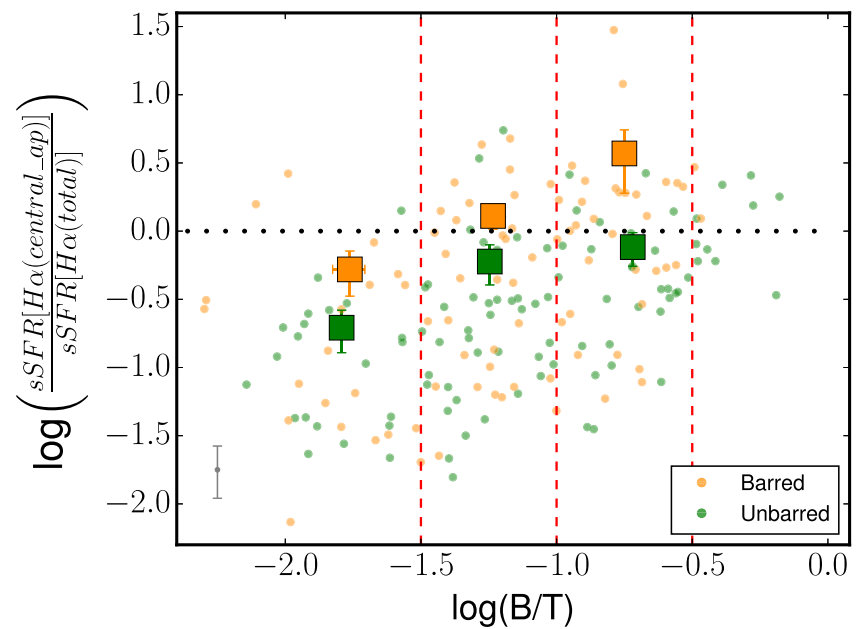

Figure 3. Variation of the $\operatorname{sSFR}[\mathrm{H} \alpha$ (central) $] / \operatorname{sSFR}[\mathrm{H} \alpha$ (total) $]$ ratio with the $\mathrm{B} / \mathrm{T}$ parameter in $g$ band. Symbols and color-coding are the same as in Figure 2.

the Sérsic index $n_{b}$ (see Fisher \& Drory 2008, 2016), where classical bulges are characterized by $n_{b}$ values greater than 2 while pseudobulges have values lower than 2 . Using the $n_{b}$ parameters derived from the 2D photometric decomposition, $72 \%$ of our bulges would be classified as pseudobulges, while the remaining $28 \%$ would appear as classical bulges.

Attending to the previous criterion, the percentages for pseudobulges in our late-type galaxies are as follows: $74 \%$ for $\mathrm{Sb}, 91 \%$ for $\mathrm{Sc}$, and $80 \%$ for $\mathrm{Sd}$ (this is due to the low number of galaxies in this bin, where four of the five galaxies are classified as pseudobulges). The median value of the $\operatorname{SFR}\left(\right.$ central $\left._{a p}\right) / \operatorname{SFR}\left(\right.$ total $\left._{a p}\right){ }^{18}$ is higher for the $\mathrm{Sb} / \mathrm{c}$ barred galaxies in comparison with unbarred galaxies. This result points out that the SFR in the central parts of these galaxies may be enhanced by the presence of a bar. Nevertheless, this trend is not found for other morphological types, perhaps due to much lower number statistics in those types.

As the majority of our galaxies are concentrated in the bin of $\mathrm{Sb} / \mathrm{c}$ objects, making the dynamic range of our morphological classification smaller, we also explore the behavior of the SFR $[\mathrm{H} \alpha$ (central) $] / \mathrm{SFR}[\mathrm{H} \alpha($ total $)]$ ratio with the $\mathrm{B} / \mathrm{T}$ parameter (Figure 2). As commented before, B/T is obtained from the $2 \mathrm{D}$ decomposition analysis and does not depend on a visual classification.

In order to quantify whether or not the presence of the bar is affecting the SFR in the bulge component, we split the sample into three bins: $\log (\mathrm{B} / \mathrm{T})<-1.5,-1.5<\log (\mathrm{B} / \mathrm{T})<-1.0$, and $-1.0<\log (\mathrm{B} / \mathrm{T})<-0.5$. Big squares represent the logarithm of the mean value for the SFR $[\mathrm{H} \alpha$ (central)]/SFR[H $\alpha$ (total)] ratios in each bin for purely star-forming galaxies, while big stars refer to galaxies that have been classified as type 2 AGNs. The 1:1 (dotted) line corresponds to the locus of galaxies that, having only bulge and disk components, would show the same extinction-corrected $\mathrm{H} \alpha$-to-optical ( $g$-band) luminosity ratio among these two components. The main result from this figure is that star-forming galaxies present higher mean SFR central values for barred galaxies (orange squares) than for unbarred ones (green squares). This effect is especially

\footnotetext{
${ }^{18}$ The ap subscript indicates that these are smooth-aperture SFR measurements as explained in Section 3.1. This subscript appears in the corresponding figures, but we have not included it throughout the text for simplicity.
} 
important for the cases of $\mathrm{B} / \mathrm{T}$ smaller than 0.1 . The enhancement of the central SFR due to the presence of bars has been pointed out by several authors using observational data (de Jong et al. 1984; Devereux 1987; Ellison et al. 2011; Wang et al. 2012; Florido et al. 2015) and also in recent dynamical simulations such as in Carles et al. (2016). As a result, a rejuvenation of the stellar populations in the center of barred galaxies has been also claimed by Fisher (2006) and Coelho \& Gadotti (2011), among others.

We can also analyze the connection between the presence of bars and AGN activity. We find that the optical bar fractions are similar for star-forming objects and type 2 AGN host galaxies, $43.9 \%$ and $52.2 \%$, respectively. This result is in accordance with previous works (Mulchaey \& Regan 1997; Hao et al. 2009). Nevertheless, galaxies hosting a type 2 AGN show less difference between the mean central SFR values for barred (orange stars) and unbarred (green stars) galaxies in comparison with purely star-forming objects. If bars and AGNs are simultaneously present, the effect of the bar in triggering the central SFR is reduced. Finally, it is also clear that type 2 AGNs are quenching the central SFR in their host galaxies, at least for small values of the $\mathrm{B} / \mathrm{T}$ parameter.

To better understand the increase in the SFR in the central parts of star-forming barred galaxies, we examine the behavior of the sSFR ( $\mathrm{sSFR}=\mathrm{SFR} / M_{\star}$ ) in fixed bins of $\mathrm{B} / \mathrm{T}$ values. Figure 3 shows that barred galaxies tend to have higher mean values of the sSFR in their central regions compared to unbarred galaxies. The horizontal dotted line here represents the location of galaxies that, having only bulge and disk components, would have the same sSFR in these two components. From this plot it is clear that low-B/T galaxies with only bulge and disk components have higher disk sSFR values than their bulges. This is possibly related to blue optical to infrared colors and the presence of significant intermediatedage stellar populations in their disks.

From this section, we can conclude that there is a clear relation between the SFR and SSFR in the central part of the galaxies and the presence of bars. Star-forming barred galaxies show higher values of their central SFR and SSFR than unbarred galaxies. This trend is present when we analyze the variation of the SFR with the $\mathrm{B} / \mathrm{T}$ ratio, while it is not as clear with the morphological type, probably due to the low statistics for early-type and $\mathrm{Sd} / \mathrm{m}$ galaxies. Besides, morphological type is also related to other aspects, such as the definition of the spiral arms or the surface brightness. In contrast, the $\mathrm{B} / \mathrm{T}$ is a more robust parameter to quantify the variation of the SFR in the central part of the galaxies, as it is related to the bulge prominence. This finding supports the idea of bars driving gas efficiently toward the central regions of galaxies, causing an enhancement of the SFR and the importance of the internal secular processes for the evolution of galaxies. On the contrary, in type 2 AGNs we do not find a significant difference in the central SFR between barred and unbarred galaxies. Thus, nuclear activity should play a role in quenching the central SFR.

\subsection{Main Sequence}

The correlation observed between SFR and stellar mass $\left(M_{*}\right)$, often referred to as the galaxy Main Sequence (MS), has been extensively studied in the local universe and at high redshift (Daddi et al. 2007; Elbaz et al. 2007, 2011; Noeske et al. 2007; Wuyts et al. 2011; Whitaker et al. 2012, 2014, 2015; Magnelli et al. 2014;
Speagle et al. 2014; Catalán-Torrecilla et al. 2015; Lee et al. 2015; Renzini \& Peng 2015; Cano-Díaz et al. 2016; Duarte Puertas et al. 2017).

Figure 4(a) shows the MS for the galaxies in our sample classified according to their morphological type. For the sake of clarity, we include here the fitting by Elbaz et al. (2007) that shows the region of the diagram where local star-forming galaxies are placed. Most of the late-type galaxies in our sample are located in this region. On the other hand, S0/SOa, $\mathrm{Sa} / \mathrm{Sab}$, and some $\mathrm{Sb} / \mathrm{Sbc}$ galaxies are comparatively less efficient at forming stars at the present time, meaning that for the same stellar mass they are placed outside the MS as shown in this diagram.

Some of the previously mentioned studies claimed that there is a turnover of the MS for stellar masses $M_{*}>10^{10} M_{\odot}$. We analyze whether or not this particular trend is also present in our sample. Instead of imposing a stellar mass cut, we divide the sample into two groups: (i) $\mathrm{Sb} / \mathrm{Sbc}$ objects and (ii) $\mathrm{Sc} / \mathrm{Scd}$ together with $\mathrm{Sd} / \mathrm{Sdm}$ galaxies. Nevertheless, this morphological type cutoff is quite similar to the one used for stellar mass, as the majority of $\mathrm{Sb} / \mathrm{Sbc}$ galaxies tend to have stellar masses larger than $10^{10} M_{\odot}$, while most of the $\mathrm{Sc} / \mathrm{Scd}$ and $\mathrm{Sd} / \mathrm{Sdm}$ objects have masses below $10^{10} M_{\odot}$. Moreover, the fact that massive late-type spirals are clearly on the MS while early-type ones of the same mass are significantly offset does advise on the use of other criteria besides mass to perform the analysis of the MS. The fittings for both cases are shown in Figure 4(a) (green and blue lines, respectively). Star-forming galaxies in Figure 4 are represented by circles, while AGN objects appear as star symbols. The fittings are only done for star-forming galaxies. There is an offset between them in the sense that $\mathrm{Sb} /$ Sbc galaxies tend to have lower SFR values for the same stellar mass. Also important is the change in the slope $(0.74 \pm 0.09$ for $\mathrm{Sc} / \mathrm{Scd} / \mathrm{Sd} / \mathrm{Sdm}, 0.63 \pm 0.12$ for $\mathrm{Sb} / \mathrm{Sbc}$ ), which goes in the direction of an extra flattening in the case of the $\mathrm{Sb} / \mathrm{Sbc}$ objects. ${ }^{19}$ As our sample does not contain highly inclined disks (due to the criteria imposed for the 2D decomposition; Section 3.2), we avoid effects that might be associated with an underestimate of the SFR, which would affect the slope and width of the MS (see Morselli et al. 2016). Therefore, we are in agreement with the authors that find a turnover of the MS, and we confirm this result for our sample. We go beyond this, as we find that it is not only mass driven but also related to the galaxy morphological type.

Although the analysis of the MS for integrated properties of galaxies is extremely valuable, we highlight the necessity of studying whether the MS is also present when galaxies are separated in their stellar structures (bulges, bars, and disks). In fact, there is a key question that still remains unsolved: do disks of galaxies that are quenched as a whole (i.e., are found away from the MS) populate the MS?

To shed some light on this issue, we analyze the "disk Main Sequence," that is, the relation between the SFR in the disk component and the stellar mass of the disk (Figure 4(b)). As we have done for the case of integrated values, we focus our attention on intermediate- to late-type galaxies. We find that the global trend for the MS is also reproduced for the case of the disks. Moreover, the fittings to the different morphological types, $\mathrm{Sb} / \mathrm{Sbc}$ and $\mathrm{Sc} / \mathrm{Scd}-\mathrm{Sd} / \mathrm{Sdm}$, show a similar behavior when compared with the integrated values. There is an offset

\footnotetext{
19 The relation given by Elbaz et al. (2007) is already tilted relative to the lines of constant sSFR.
} 

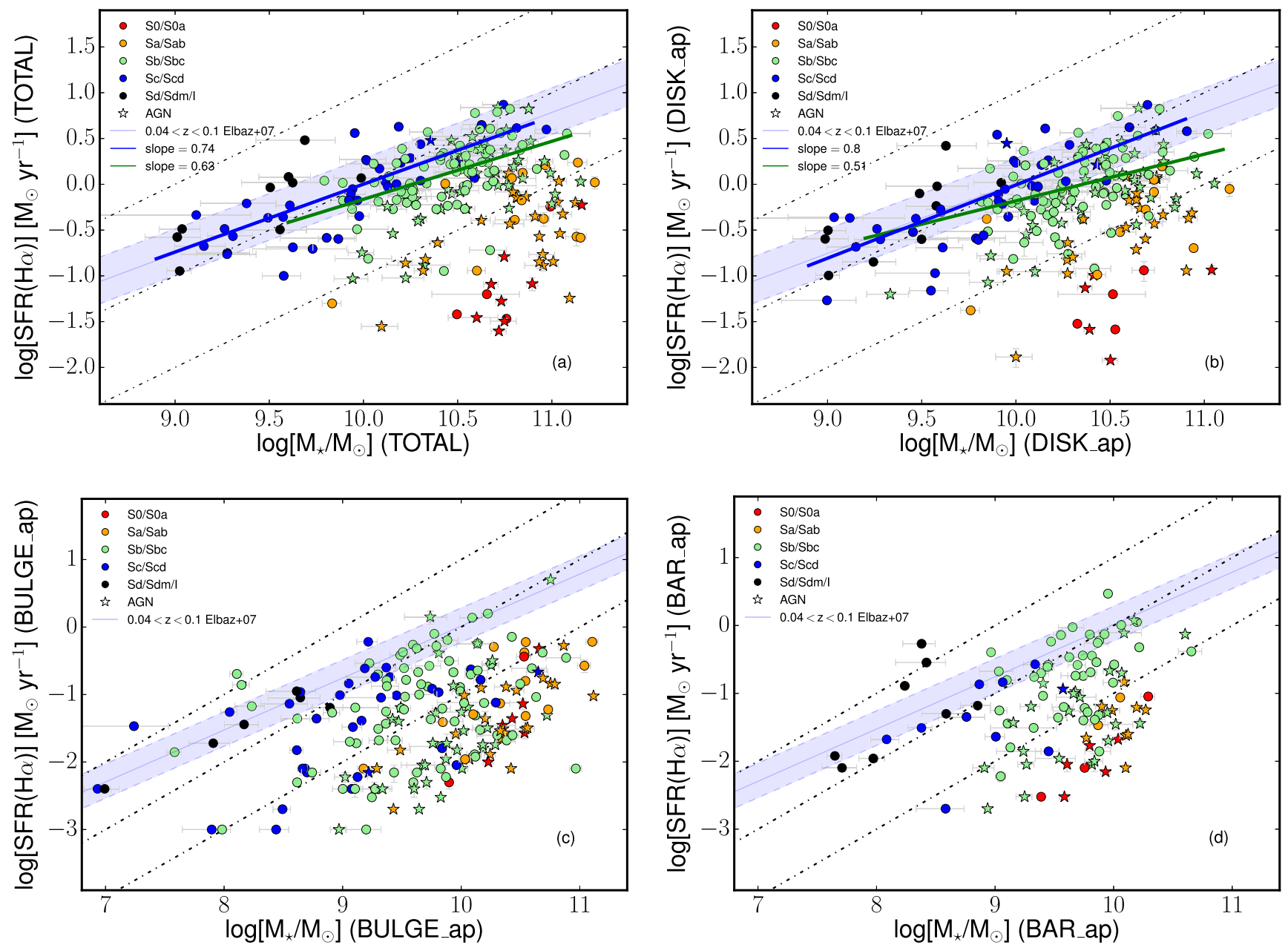

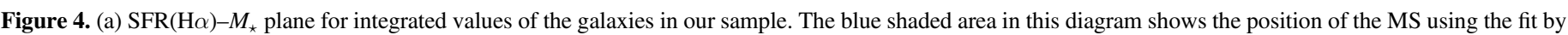

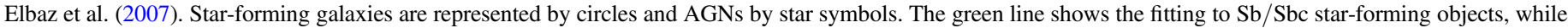

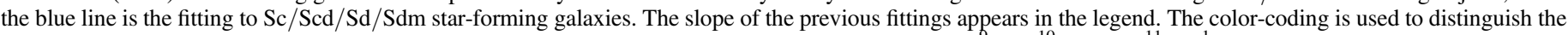

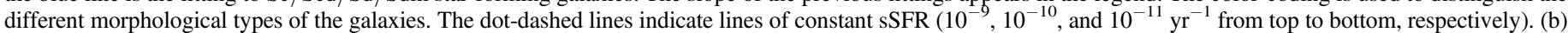

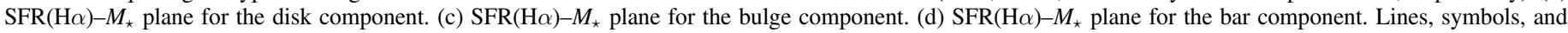
colors in panels (b), (c), and (d) are the same as in panel (a). The ap subscript in the labels indicates that these are (smooth-)aperture measurements.

between both fits, and the slope is also steeper for Sc/Scd-Sd/ $\mathrm{Sdm}$ galaxies $(0.80 \pm 0.10)$ compared to $\mathrm{Sb} / \mathrm{Sbc}(0.51 \pm 0.14)$. Then, we conclude that the current-to-past SFR has decreased in more massive disks and in earlier-type spirals relative to less massive and later-type systems. Not only entire galaxies but also disks in more massive systems have been more efficiently quenched. We note here that the dynamical range for stellar masses is quite similar for disks and for integrated galaxies, so when we refer to more massive systems, in general, we are referring to more massive disks as well. We find in the same figure that many of the disks, mainly S0/S0a and Sa/Sab, are still away from the MS on their own.

The position of the bulges in the SFR(bulge) $-M_{\star}$ (bulge) plane is shown in Figure 4(c), while for the case of the bars the SFR(bar) $-M_{\star}$ (bar) plane is shown in panel (d) of the same figure. Bulges and bars are clearly much less efficient than disks in terms of their SFR, even less if we take into account that in type 2 AGNs some of the SFR associated with the central components might not be related to recent star formation.

Until now we have shown the SFR trend of each galaxy component with their corresponding stellar mass (bulges, bars, and disks). Now, we focus on the analysis of the SFR of each component with the total galaxy stellar mass instead. The top panel in Figure 5 shows the trends for the variation of the SFR in the bulge, bar, and disk component in bins of $0.5 \mathrm{dex}$ in stellar mass. We have combined at the same time all the morphological types for each component (which will obviously increase the dispersion, as early-type spirals have lower values of their SFR, especially for the disk component). It can be seen from this figure that most of the actual SFRs in galaxies are located in the disk component, as is expected, while bars and bulges show a smaller contribution for a fixed stellar mass. As seen previously for the disks, not only with morphological type but also with stellar mass there is a clear decrease in the SFR for more massive disk galaxies (i.e., more massive systems in general, due to the similar range in total and disk stellar masses).

To conclude, we have demonstrated in this section that more massive star-forming disks and earlier-type spiral disks show a higher level of quenching. Previous studies have shown that more massive star-forming galaxies (understanding galaxies as entire systems) tend to be less efficient at forming new stars. Here, the important fact is that we treat disks as separate components of the galaxies. 

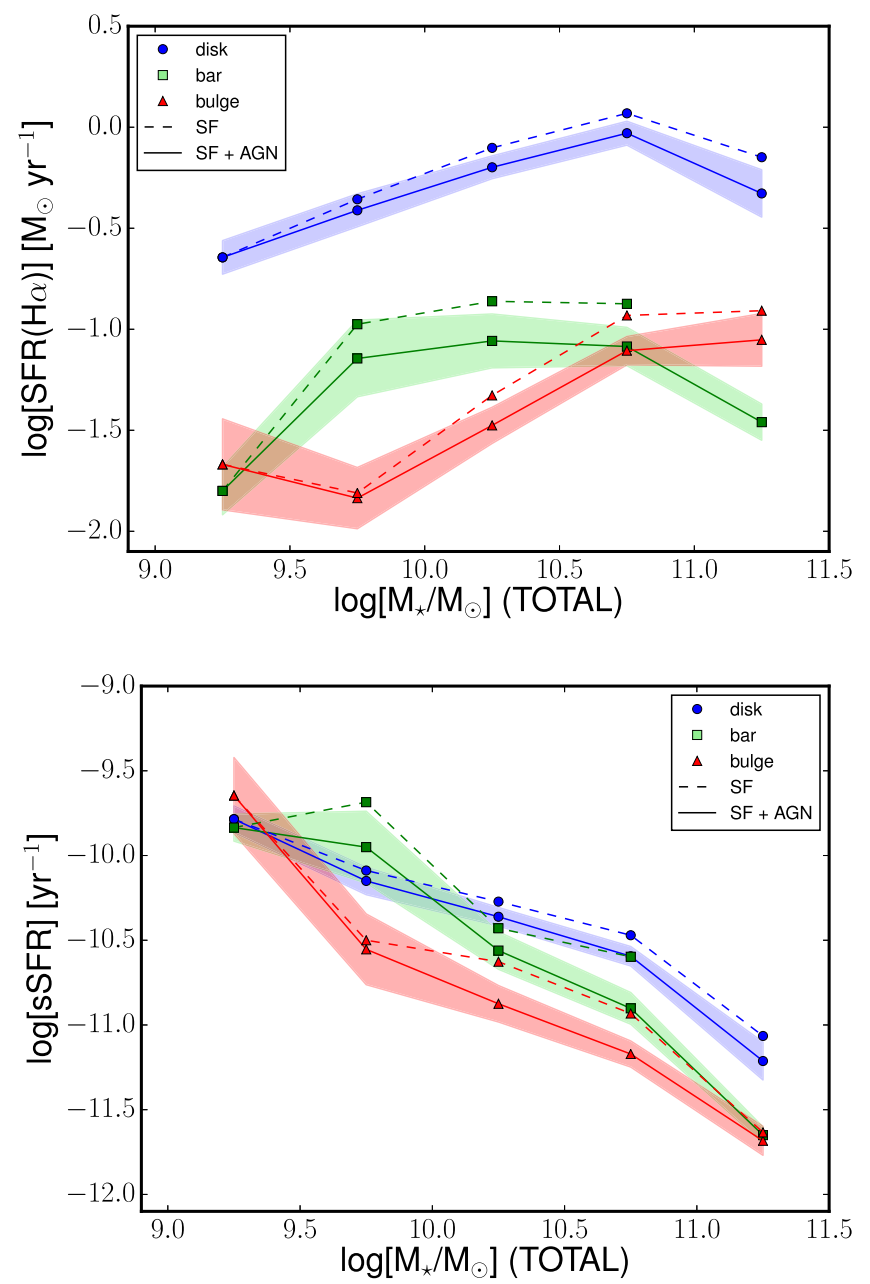

Figure 5. Top panel: variation of the $\mathrm{H} \alpha$-based SFR for the different morphological components of the galaxies (bulge, bar, and disk) with the total stellar mass of the galaxy. The trends for bulges, bars, and disks are shown in red triangles, green squares, and blue circles, respectively. Filled contours represent the $1 \sigma$ dispersion around the mean value expressed as a red, green, and blue solid line for the bulge, bar, and disk, respectively. Dashed lines show the trends just for the star-forming galaxies. Bottom panel: same as the top panel, but for the sSFR.

\section{3. $s$ SFR $-M_{\star}$ Relation for Bulges and Disks: A Clue for the Quenching of Massive Systems}

In the previous section, we have explored in detail the MS and the fact that the same relation that applies to star-forming galaxies as a whole is also valid for the disk component of the same galaxies. More surprisingly, however, galaxies that are offset from the MS have disks that are also forming stars at present at a lower rate than in the past compared to MS galaxies (i.e., they do not fall on the MS), so the position of galaxies relative to the MS is due not only to a larger contribution of the bulge component but also to a decrease in the recent SFR of their disks normalized to their mass (see below). This is true even for well-defined $\mathrm{Sb} / \mathrm{Sbc}$ spirals. In other words, there is what we have called the "disk MS," and galaxies that are away from the MS have disks that are also away from the MS. To associate this finding with the capacity for galaxies to form stars at the present time (compared with that in the past), we analyze here the specific SFR of both bulges and disks as a function of the galaxy total stellar mass. Ultimately, we aim to answer the following question: what are the mechanisms responsible for the quenching of the most luminous and massive galaxies and their disks?

Abramson et al. (2014) proposed that sSFR(disk) is approximately constant with stellar mass for $M_{\star}>10^{10} M_{\odot}$ and $\mathrm{B} / \mathrm{T}<0.6$. The authors assume sSFR(disk) $=\mathrm{SFR}$ (total) $/$ $M_{\star}($ disk) and that nuclear and bulge regions might have small contributions to the star formation. If this is the case, the growth of bulges may be the potential cause to create the flattening in the MS for the higher stellar masses. Nevertheless, we argue here that bulges also contribute to the SFR in those galaxies with higher values of their stellar masses (as shown in the previous section). Figure 6 shows the relation of the sSFR (bulge) and sSFR(disk + bar) with the total stellar mass for the galaxies in our sample. From this figure, we conclude that sSFR(disk + bar) is not constant with stellar mass, meaning that disks are not equally active at forming stars in terms of their sSFR. Besides, from the left panel in Figure 6 it can be seen that the sSFR(bulge) spans a wide range (more than 2 dex) of values and that the most active bulges present a nonnegligible value of their sSFR. As commented in Section 4.1, attending to the $n_{b}$ parameter, $72 \%$ of our bulges would be classified as pseudobulges, while the remaining $28 \%$ would appear as classical bulges. Fisher \& Drory (2016) established that bulges should be forming stars actively for sSFR $>10^{-11}$ $\mathrm{yr}^{-1}$ (typically pseudobulges), while they might be either pseudobulges or classical bulges for lower values of the sSFR. We find a median value of $1.7 \times 10^{-11}\left(8.9 \times 10^{-12}\right) \mathrm{yr}^{-1}$ for pseudobulges (classical bulges). Determining whether or not sSFR provides an accurate separation between bulges or pseudobulges is beyond the scope of this paper and would require high-resolution imaging of the nuclear regions, which is not available for the vast majority of the galaxies in our sample.

Another potential mechanism to quench the star formation of the more massive galaxies could be the presence of an AGN. Although many studies include only galaxies that are strictly star-forming, we also include here type 2 AGNs to study their relative position in the SSFR-stellar mass plane. The power of IFS data will certainly help us to resolve whether or not the presence of AGNs contribute to the quenching of the massive galaxies. We recently reported in Catalán-Torrecilla et al. (2015, Figure 19) that AGNs might have an impact on suppressing the total SFR in their host galaxies. Other works corroborate the idea of the suppression of the star formation by AGNs in the host galaxies (Shimizu et al. 2015; Leslie et al. 2016). In this section, we investigate the role of AGNs in the quenching of the SFR, not only in global terms but also in their bulges and disks separately. This is particularly important considering that, as shown above, galaxies that are away from the MS host disks that have their star formation depressed/ suppressed, so AGN quenching should thus work at galacticwide scales. The alternative is that AGN quenching is not the dominant mechanism but is coeval with another mechanism(s) that has an impact on the star formation at those scales. One possibility is the removal of a fraction of the high angular momentum gas of the disks due to interactions toward the nucleus (leading to an AGN) becoming unavailable for star formation in the disk component.

To investigate this possibility, we examine the sSFR-stellar mass plane shown in Figure 6 for bulges and disks, separately. Some interesting results emerge from these plots. First, type 2 AGNs are not homogeneously distributed in the plane. They tend to be in the high-mass end. Indeed, type 2 AGNs are mostly 

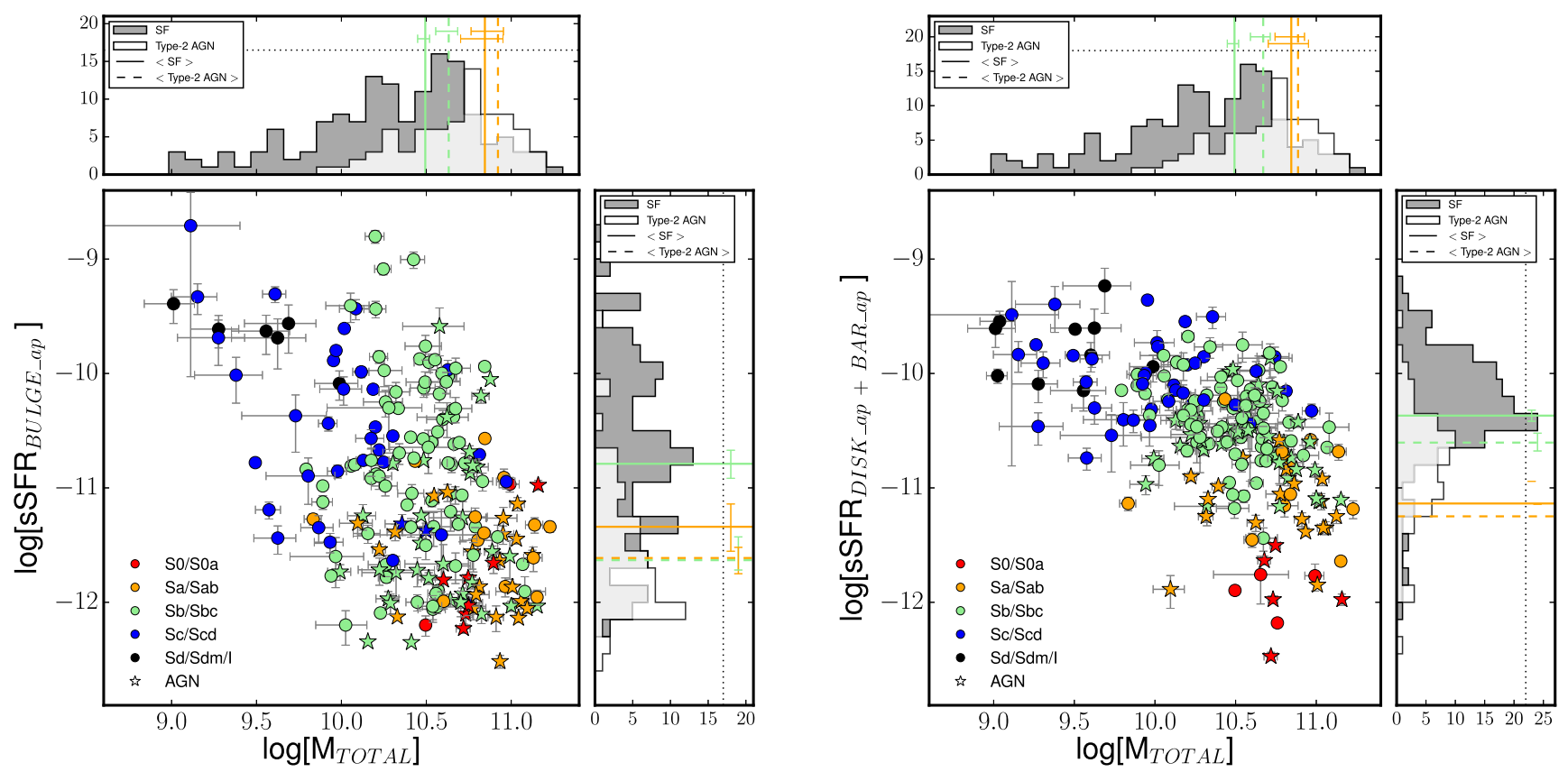

Figure 6. Left panel: sSFR for the aperture of the bulge component as a function of the total stellar mass. The circles refer to the star-forming galaxies in our sample, while the stars indicate the AGNs. The color of each symbol indicates the corresponding morphological type of the galaxy. The top histogram shows the distribution of the bulges with the total stellar mass of the corresponding galaxy that could be classified as star-forming or type 2 AGN. Vertical solid (dashed) lines represent the median value of SF (AGN) galaxies, while the errors bars are computed using the standard error of the median, i.e., $1.253 \times \sigma /$ sqrt(N), where $\sigma$ is the interval that contains $68 \%$ of the points. $\mathrm{Sb} / \mathrm{Sbc}(\mathrm{Sa} / \mathrm{Sab}$ ) types appear in light green (orange). The right histogram shows the distribution of the galaxies as a function of the sSFR in the bulge component. Vertical lines represent median values and their errors. Right panel: same as the left panel, but for the aperture of the disk+bar component.

found in galaxies with stellar mass values in the range of $10^{10}-10^{11.5} M_{\odot}$ (white histograms on the top of both panels in Figure 6). We also find that there is a clear decrease in the sSFR values when a type 2 AGN is present. Bulges of AGN hosts show a median SSFR(bulge) that is 0.89 dex below that of starforming galaxies when the difference in median stellar mass is +0.32 dex. For the case of the disks there is a $-0.52 \mathrm{dex}$ difference in median SSFR(disk) and +0.41 dex in median mass. Nevertheless, it is important to quantify whether this effect is still present in terms of the same morphological type or not. Thus, due to the lack of type 2 AGNs in most of our late-type galaxies, in agreement with previous works (Moles et al. 1995), we restrict the following analysis to $\mathrm{Sa} / \mathrm{Sab}$ and $\mathrm{Sb} / \mathrm{Sbc}$ objects. Bulges of $\mathrm{Sa} / \mathrm{Sab}(\mathrm{Sb} / \mathrm{Sbc})$ show a median sSFR that is $0.27(0.84)$ dex below that of star-forming galaxies, while the difference in the median value of the stellar mass is $0.08(0.14)$ dex. For the case of the disks, $\mathrm{Sa} / \mathrm{Sab}(\mathrm{Sb} / \mathrm{Sbc})$ galaxies exhibit a difference in the median values of sSFR for star-forming galaxies and AGNs of $0.11(0.23)$ dex, while the difference in stellar masses is 0.04 (0.16) dex (solid and dashed vertical lines in the top and right histograms of the right panel of Figure 6). If the bars are excluded, the median values of sSFR for star-forming galaxies and AGNs are $0.13(0.20)$ dex for $\mathrm{Sa} / \mathrm{Sab}(\mathrm{Sb} / \mathrm{Sbc})$. The previous results suggest a possible damping of the SFR in both components (bulges and disks) due to the presence of AGNs. We prefer the term "damping" here as compared to "quenching." It is not clear whether this decrease in the sSFR is enough (nor if it lasts long enough) to make these galaxies evolve toward and remain in the red sequence, something for which galaxy evolution models require a strong quenching of the star formation in massive galaxies at high redshift (Weinberger et al. 2017, and references therein). Also, we find that bulges show a constant decline of the sSFR across the entire stellar mass range. On the contrary, the decrease in the disk component is more dramatic when galaxies reach a certain stellar mass, typically around $10^{10.5} M_{\odot}$. Finally, a significant trend with the morphological type is also found. Late-type galaxies have higher values of their SSFR for both components, bulges and disks.

To clarify the previous trends, the bottom panel of Figure 5 shows the variation of the sSFR in the different morphological components (bulge, bar, and disk) in bins of $0.5 \mathrm{dex}$ in total stellar mass. As done previously in the case of the SFR (top panel in the same figure), all the morphological types for each component are combined at the same time (spreading the dispersion as early-type spirals present lower sSFR values). Again, it is clear from this figure that the disk component is significantly more effective than the bulge at forming new stars, especially for $M_{\star}>10^{9.5} M_{\odot}$, and the steeper decline for the bulges at the lower stellar mass bin.

From the results in this section, we conclude that the presence of an AGN might be linked with some level of the damping of the SFR in both the bulge and the disk component even in the local universe. For both cases, the sSFR decreases when an AGN is present, this effect being higher for the bulges in competition with the effect of the bars. We identify the same behavior among different morphological types such as $\mathrm{Sa} / \mathrm{Sab}$ and $\mathrm{Sb} / \mathrm{Sbc}$. Again, due to the short timescale traced by the $\mathrm{H} \alpha$ line emission, we cannot infer whether the AGN phase is cause, consequence, or coeval with the star formation quenching/damping process. Besides, as discussed in Section 3.5, we cannot exclude that a fraction of these low-luminosity AGNs could be powered by hot evolved stars in regions with basically null recent star formation.

\subsection{Relation with Other Parameters}

As discussed previously in Sections 4.2 and 4.3, stellar mass seems to be the main driver of the star formation, and, after it, AGN activity also plays an important role. Nevertheless, it is 
worth exploring the role of other (possibly secondary) parameters that are known to either trigger or quench star formation. In that regard, the following subsections aim to shed some light on the effect that stellar kinematics and the environment have on the star formation processes taking place in our sample.

\subsubsection{Stellar Kinematics}

In this section we explore how stellar kinematics could regulate the star formation in the inner regions of our galaxies. With this aim in mind, we analyze the line-of-sight (LOS) stellar velocity dispersions for the bulge component. We have restricted the analysis of the LOS velocity dispersions to the bulge component, due to the fact that the LOS velocity dispersion distribution for each component (bulge, bar, and disk) is quite distinct in the regions where they coexist. This case is especially important for the internal parts of the disks, where the values could be affected by the bulge contamination, as this component tends to be the more prominent there. Thus, measuring the stellar velocity dispersion of only the disk component presents intrinsic limitations. For the previous reason, we will focus here on the possible impact of the stellar velocity dispersion in bulges on their SFR.

We employ the CALIFA stellar velocity dispersion maps created by Falcón-Barroso et al. (2016) using V1200 grating data. In order to calculate the integrate velocity dispersions for the bulge, we first multiply the stellar velocity dispersion map by the luminosity-weight map of the bulge component in the $g$ band (previously derived as explained in Section 3.2). Then, we divide it by the $g$-band luminosity taking into account only those pixels where the dispersion values are greater than zero. The method applied to obtain the luminosity-weight maps is the same as the one explained in Section 3.2. The only difference is that here we used Voronoi bins instead of spaxels, as each Voronoi bin provides its own velocity dispersion for the stars. Thus, the expression used to obtain the LOS stellar velocity dispersion for each bulge component is the following:

$$
\sigma_{\text {bulge }}=\frac{\sum_{i=1}^{N} F_{i} \cdot \sigma_{i}}{\sum_{i=1}^{N} F_{i}},
$$

where the $i$ subscript refers to the Voronoi bin used in each case. The values of the $\sigma_{\text {bulge }}$ calculated in this section are given in Table 1. The methodology followed in this work is similar to other kinematical parameters based on 2D spectroscopic data used in the literature (see, e.g., Emsellem et al. 2011 , for a similar recipe for $\lambda_{\mathrm{R}}$ ), and it is easily reproducible by other authors using data from different instruments. Moreover, it allows us to go beyond the standard method, as we apply the luminosity-weight maps for the bulge component, which should restrict in a better way the calculation of the LOS velocity dispersions.

In Figure 7 (top panel) we show the relation between the SFR in bulges and the stellar velocity dispersions computed as in Equation (4). The sample is separated by spectral class (AGN and SF). We find that for the same LOS velocity dispersion, star-forming galaxies show higher bulge SFRs than those of AGN hosts. This, in principle, might be simply due to the correlation between stellar mass and stellar velocity dispersion found in ellipticals and bulges (Faber \& Jackson 1976; Chilingarian et al. 2008; Falcón-Barroso et al. 2011) and
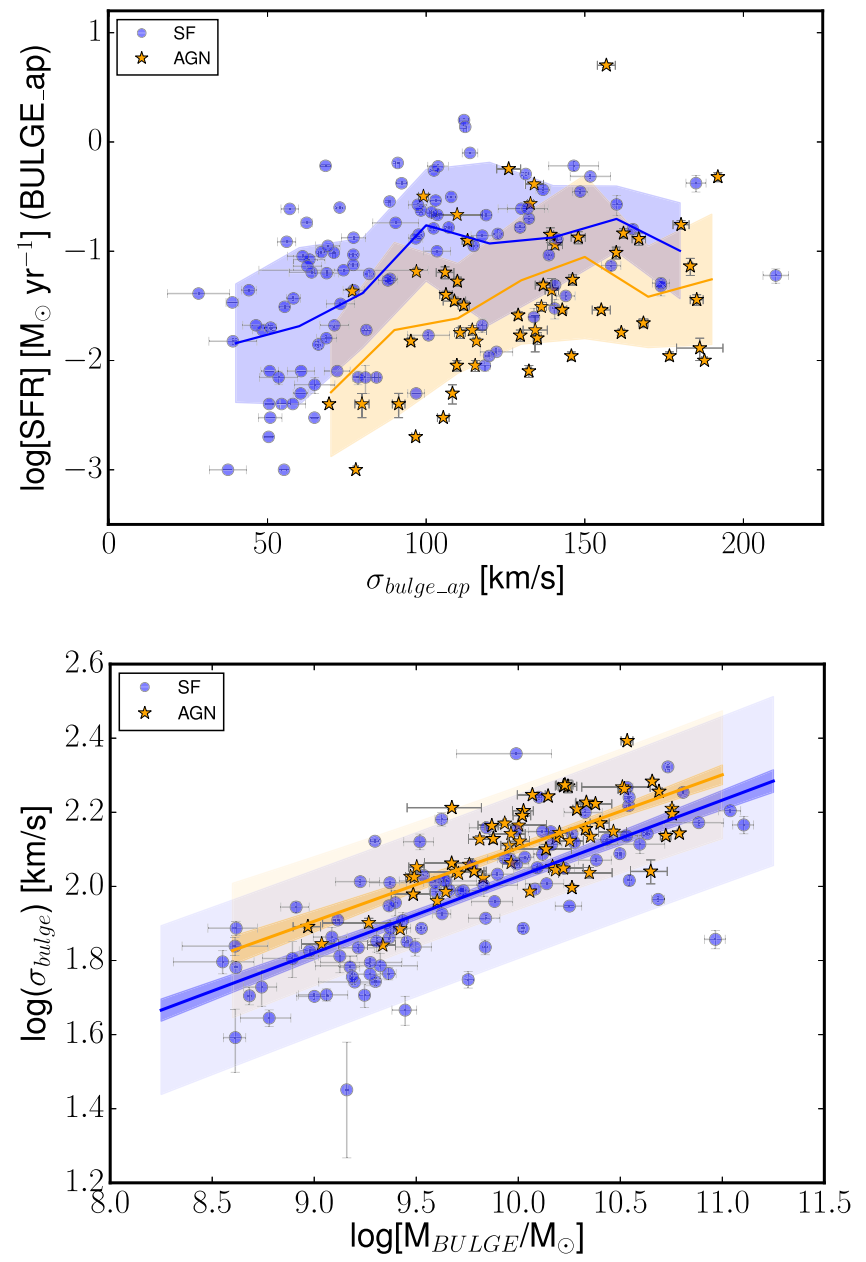

Figure 7. Top panel: SFR in the aperture of the bulge component vs. LOS stellar velocity dispersion. Galaxies are plotted using different colors and symbols, blue circles for star-forming objects and orange stars for type 2 AGNs. Blue (orange) filled contours represent the $1 \sigma$ dispersion around the mean value shown as a blue (orange) solid line for SF (type 2 AGN) galaxies. Bottom panel: Faber-Jackson relation for the bulges in our sample. The blue (orange) solid line shows the best fitting for SF (AGN) galaxies. Dark-shaded areas correspond to the error bands of the fittings when only errors associated with slope and intercept are taken into account. Light-shaded areas mark the global uncertainty bands once an additional $s$ parameter that takes into account the intrinsic variation of the points is also included.

the noisy correlation between the former and the SFR (see Figure 4(c)).

Additionally, a higher $\sigma_{\text {bulge }}$ (even for the same stellar mass) could also contribute to dynamically heating the gas and to reducing the efficiency of star formation. In order to test whether both effects (or only the stellar mass) are at play, we compare the $\sigma_{\text {bulge }}$ and stellar mass values of our bulges in Figure 7 (bottom panel). The blue (orange) solid line shows the best fitting for SF (AGN) galaxies. We have employed the Markov chain Monte Carlo method to sample the probability density function of our model parameters. The Pymc3 code (Salvatier et al. 2016) is used to implement the analysis. The slope and intercept of a line are computed considering uncertainties in both axes. Also, an additional $s$ parameter that takes into account intrinsic variations of the individual points is included. The best fitting for star-forming galaxies is $-0.035( \pm 0.180)+0.206( \pm 0.018) \times$ $\log \left[M_{\mathrm{BULGE}} / M_{\odot}\right]$, with $s=0.110 \pm 0.009$, while for the AGNs it is $0.130( \pm 0.253)+0.197( \pm 0.025) \times \log \left[M_{\mathrm{BULGE}} / M_{\odot}\right]$, with $s=0.082 \pm 0.008$. A similar value for the slope in 
both cases is found, while there is a slightly higher value for the intercept of AGNs. This indicates higher $\sigma_{\text {bulge }}$ values for the AGNs at stellar masses larger than $10^{9.5} M_{\text {bulge }} / M_{\odot}$ (the bulge stellar mass range where most of the SF and AGNs coexist). The dark-shaded area corresponds to the error bands of the fitting when only errors associated with slope and intercept are taken into account. The light-shaded area marks the global uncertainty bands once the additional $s$ is also included. If we fix the slope of the fits to both data sets to $1 / 4$, then the mean difference in $\sigma_{\text {bulge }}$ between the two samples would be 0.03 dex. This Faber-Jackson relation shows that even for the same stellar mass, star-forming galaxies tend to have a lower $\sigma_{\text {bulge, suggesting that a }}$ dynamically cooler stellar population in the bulges can more easily host star formation.

\subsubsection{Environment}

Environment is another parameter that can strongly affect the SFR and further stellar mass growth of galaxies and components within galaxies. It is also thought to be the cause of the well-known morphology-density relation (Dressler 1980). The three main broad mechanisms proposed to play a role in this sense are mergers/interactions (sometimes referred to as galaxy harassment), ram pressure and viscous stripping of cold gas, and strangulation in the supply of warm/hot gas (see Kawata \& Mulchaey 2008). As these mechanisms act differently in different regions of galaxies and on different timescales, the study of the distribution of the current SFR is key to determining whether or not they are contributing on specific objects and which one dominates in each case (Boselli \& Gavazzi 2006). Moreover, in the case of mergers and interactions, they might lead to either quenching or triggering of the star formation depending on the type of interaction (mass ratios, impact parameters) and on the region considered (nuclear regions, outer disks, or even tidal tails). Thus, to investigate whether or not the environment is playing a significant role on the SFR or sSFR of the different structural components of our galaxies, we use the local density values from the projected comoving distance to the fifth-nearest neighbor of the target galaxy. The projected galaxy density, $\Sigma_{5}$, in number of galaxies per $\mathrm{Mpc}^{2}$ is calculated as

$$
\Sigma_{5}=\frac{N}{\pi\left(d_{5}\right)^{2}} .
$$

We have reliable measurements for a total of 140 objects, while we lack $\Sigma_{5}$ measurements for 87 galaxies (see Table 1). This is mainly because the area enclosing the nearest neighbor lies outside the footprint of the SDSS. This means that for these galaxies we cannot obtain a reliable measurement of the density, since we do not know whether there is another close galaxy outside the survey area.

In Figure 8 (top panels), we present the variation of the $\mathrm{H} \alpha$ based SFR in the disk and in the bulge components as a function of galaxy density, $\Sigma_{5}$. We appreciate a weak trend between both parameters. Galaxies tend to have lower values of their SFR in both components (bulges and disks) for higher values of the galaxy density. These $\Sigma_{5}$ values are associated with medium- and high-density environments, although the latter case is not well sampled owing to a lack of galaxies in this position of the diagram. The previous trend is consistent with other works that used galaxy density to estimate environmental effects associated with SFR but using integrated values (e.g., Gómez et al. 2003) and with high-resolution cosmological simulations that show a reduction of the SFR in high-density environments at $z=0$ (Tonnesen \& Cen 2014).

In order to properly assess this effect, which is also related to the mass of the galaxies, the bottom panels of Figure 8 represent the relationship between sSFR (sSFR(disk) and sSFR (bulge)) and galaxy density. The evidence for a decrease in the SFR and sSFR in bulges and disks with the presence of type 2 AGNs has been already discussed in Sections 4.2 and 4.3. We will focus here on the case of the SFR and SSFR measured in the disks of star-forming galaxies (left panels), as the trends found for bulges are clearly more noisy, albeit having similar slopes. It is clear that disks in star-forming galaxies with intermediate to high values of $\Sigma_{5}$ show higher sSFR values. The analysis of the morphological types that are responsible for the increase in the sSFR at intermediate densities (groups) indicates that this is due to a larger number of $\mathrm{Sd}$ (or later) galaxies being found in groups than in the field for our galaxy sample. The number of galaxies when split by environment and type is not large enough to drive firm conclusions. Despite that fact, an enhancement in the disk star formation activity for galaxies located in groups could increase the number of these objects in the sample, either due to a positive bias toward actively star-forming systems being included in CALIFA or by means of a morphological transformation toward later types.

\section{Conclusions}

The uniqueness of combining IFS data and a $2 \mathrm{D}$ multicomponent photometric decomposition makes it possible to disentangle the distribution of the extinction-corrected $\mathrm{H} \alpha$ based SFR within different stellar structures in galaxies (bulges, bars, and disks). It also allows us to determine how these morphological components would grow in stellar mass owing to in situ star formation. With this aim in mind, we have analyzed which mechanisms might either trigger or quench the star formation in a sample of 219 CALIFA nearby galaxies.

This work led to the following main conclusions.

1. There is an enhancement of the central SFR and SSFR due to the presence of bars for star-forming galaxies, in agreement with the results found in previous works (de Jong et al. 1984; Devereux 1987; Ellison et al. 2011; Wang et al. 2012; Florido et al. 2015). This finding supports the idea that gas might be funneled into the central part of the galaxies triggering the star forming processes. On the other hand, this effect is reduced when a type 2 AGN is present, making the SFR values in barred and unbarred galaxies more similar between them in terms of SFR (Section 4.1).

2. We examine the SFR $-M_{\star}$ plane focusing on the starforming Main Sequence, treating galaxies as entire systems and also analyzing this sequence for their basic stellar structures (bulges, bars, and disks). The results indicate that there is a turnover in the MS not only for integrated values but also for disks, i.e., in the correlation between the SFR(disk) and the $M_{\star}$ (disk). This fact means that also the disks of massive galaxies have been more efficiently quenched than their lower-mass counterparts (Section 4.2).

3. The correlation between sSFR in the stellar components of the galaxies (bulge, bar, and disk) and the total stellar mass is analyzed to identify which mechanism(s) might be damping the star formation in more massive systems. 

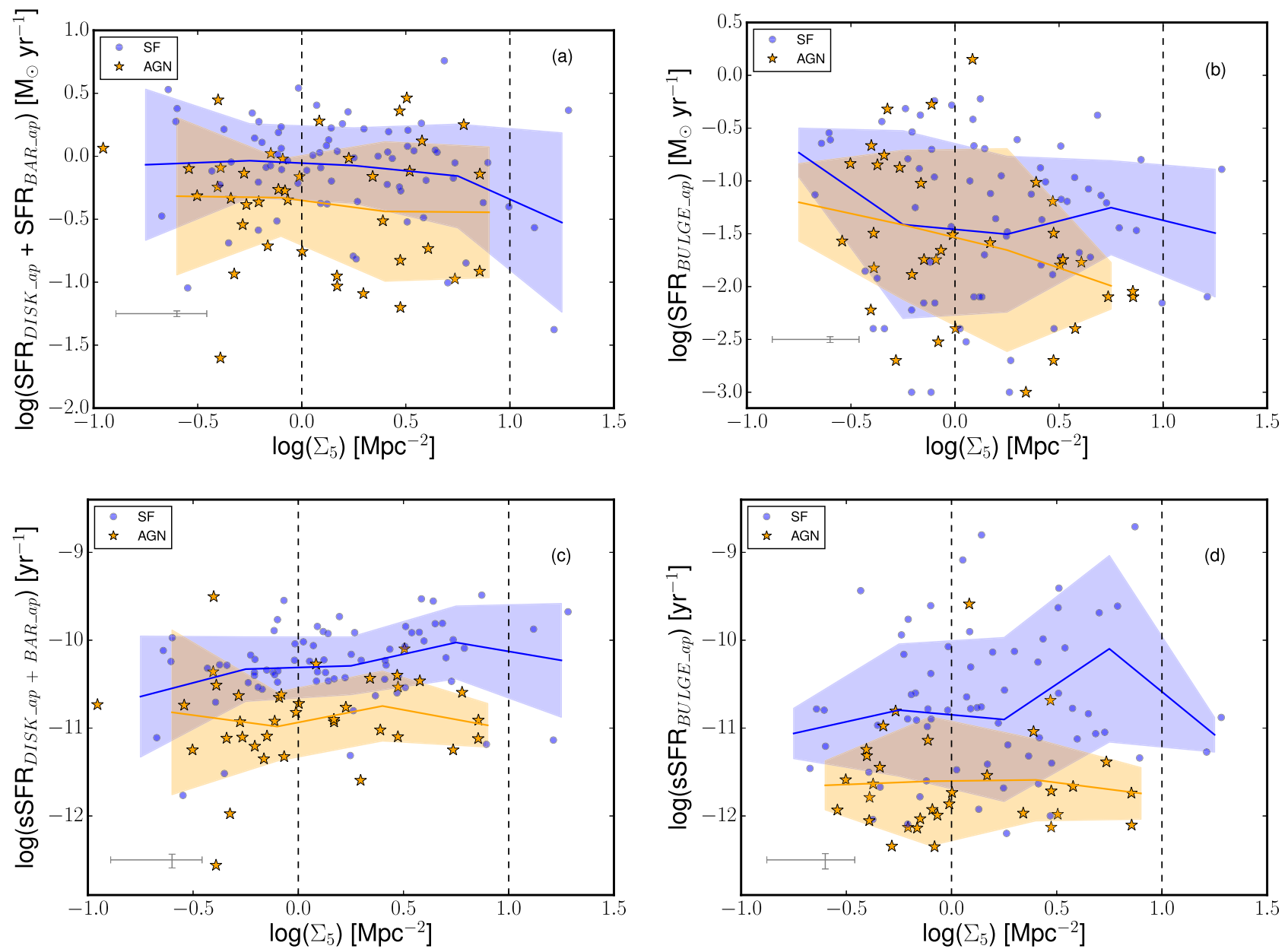

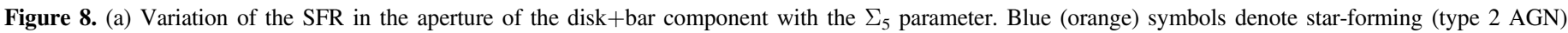
galaxies. Blue (orange) filled contours represent the $1 \sigma$ dispersion around the mean value expressed as a blue (orange) solid line for the star-forming (type 2 AGN) galaxies. Vertical dashed lines correspond to $\log \left(\Sigma_{5}\right)=0.0$ and $\log \left(\Sigma_{5}\right)=1.0$, i.e., the demarcation between low- to medium-density and medium- to high-density environments, respectively. (b) Same as panel (a), but for the SFR in the bulge component. (c) Variation of the sSFR in the disk+bar component as a function of the $\Sigma_{5}$ parameter. Color-coding and symbols are the same as in previous panels. (d) sSFR in the bulge component as a function of the $\Sigma_{5}$ value. Same color-coding and symbols as in previous panels.

First, we observe a decline associated with the sSFR (bulge) that is present across the entire stellar mass range, while in the case of the sSFR(disk+bar) the decrease becomes significant for $M_{\star}>10^{10.5} M_{\odot}$. We also find that galaxies hosting a type 2 AGN tend to have lower values of their sSFR in both bulges and disks, separately. We previously reported this behavior for entire systems in Catalán-Torrecilla et al. (2015). This effect is more important for the case of the bulge component in comparison with the disk component, $-0.89(-0.52)$ dex lower in the median value of the sSFR bulge (disk+bar) and $+0.32(+0.41)$ dex more massive in terms of the median value of the total stellar mass. As type 2 AGNs tend to be in the more massive systems in our sample, $10^{10}-10^{11.5} M_{\odot}$, we analyze whether this trend is also present in terms of the morphological type. We restrict the analysis to the more abundant objects with these morphological types and stellar masses, i.e., $\mathrm{Sa} / \mathrm{Sab}$ and $\mathrm{Sb} / \mathrm{Sbc}$ objects. Bulges of $\mathrm{Sa} / \mathrm{Sab}(\mathrm{Sb} / \mathrm{Sbc}$ ) show a median sSFR that is $0.27(0.84)$ dex below that of starforming galaxies, while the difference in the median value of the stellar mass is $0.08(0.14)$ dex. For the case of the disks, $\mathrm{Sa} / \mathrm{Sab}(\mathrm{Sb} / \mathrm{Sbc})$ galaxies exhibit a difference in the median values of sSFR for star-forming galaxies and AGNs of $0.11(0.23)$ dex, while the difference in stellar masses is $0.04(0.16)$ dex (Section 4.3).

4. The previous point supports the idea of negative feedback produced by type 2 AGN galaxies. We cannot exclude, however, that other possibilities might be at a play. On one hand, at least a fraction of the LLAGNs that are classified as LINERs could be powered by hot evolved stars. In those cases, the low SFR and sSFR values derived would indicate that these galaxies define a lower photoionization envelope (i.e., a minimum $\mathrm{EW}_{\mathrm{H} \alpha}$ ) associated with evolved (non-star-forming) stellar populations in very massive systems (Cid Fernandes et al. 2011). Thus, for these galaxies mass would be solely the parameter driving the level of current SFR in galaxies and in components within galaxies. On the other hand, AGN damping might be coeval with another mechanism(s) that is (are) regulating the star formation processes (Section 4.3).

5. The role that stellar kinematics could have in regulating the star formation processes is analyzed by means of the 
light-weighted LOS stellar velocity dispersion of the bulge component, $\sigma_{\text {bulge. }}$ Type 2 AGN galaxies show higher values of the $\sigma_{\text {bulge }}$ than star-forming objects. This bimodality is also displayed in the Faber-Jackson relation, where type 2 AGN galaxies present higher values of the $\sigma_{\text {bulge }}$ for the same stellar mass than starforming objects (Section 4.4.1).

6. The effect that environment has on the star formation processes is studied using the projected galaxy density, $\Sigma_{5}$. We find that galaxies have lower values of the SFR in both bulges and disks when they are located in intermediateand high-density environments (Section 4.4.2).

In brief, this study concludes that the parameter that is affecting more strongly the current SFR of a galaxy, even the SFR associated with their basic stellar structures, is the stellar mass. Star formation damping by type 2 AGNs also plays a significant role for bulges, while this effect is less efficient in disks. Nevertheless, we do not discard the possibility that AGNs might be coeval with other processes affecting the star formation processes in the galaxies. In addition to the stellar mass and the nuclear activity, it seems that kinematics and environment act as secondary parameters in regulating the SFR, at least in our sample of galaxies. We emphasize the importance of applying 2D multicomponent photometry decomposition over IFS data to understand the role that different mechanisms play in quenching or triggering the star formation in the structural components that form galaxies.

This study makes uses of the data provided by the Calar Alto Legacy Integral Field Area (CALIFA) survey (http://califa. caha.es). CALIFA is the first legacy survey being performed at Calar Alto. The CALIFA Collaboration would like to thank the IAA-CSIC and MPIA-MPG as major partners of the observatory, and CAHA itself, for the unique access to telescope time and support in manpower and infrastructures. The CALIFA Collaboration thanks also the CAHA staff for the dedication to this project. We would like to thank A. Aragón-Salamanca for useful comments and suggestions. C.C.-T. gratefully acknowledges the support of the Spanish Ministerio de Educación, Cultura y Deporte by means of the FPU Fellowship Program and the Postdoctoral Fellowship of the Youth Employment Initiative (YEI) European Program. The authors also acknowledge the support from the Plan Nacional de Investigación y Desarrollo funding programs, AYA2012-30717 and AyA201346724P, of Spanish Ministerio de Economía y Competitividad (MINECO).

\section{ORCID iDs}

C. Catalán-Torrecilla (1) https://orcid.org/0000-0002-8067-0164

A. Gil de Paz (1) https://orcid.org/0000-0001-6150-2854

A. Castillo-Morales (1) https://orcid.org/0000-0003-4964-3245

J. Méndez-Abreu (1) https://orcid.org/0000-0002-8766-2597

E. Florido (ㄴ) https://orcid.org/0000-0002-2982-9424

R. García-Benito (1) https://orcid.org/0000-0002-7077-308X

B. Husemann (10 https://orcid.org/0000-0003-2901-6842

J. Iglesias-Páramo (i) https://orcid.org/0000-0003-2726-6370

D. Mast (1) https://orcid.org/0000-0003-0469-3193

S. Pascual $\odot$ https://orcid.org/0000-0002-9351-6051

T. Ruiz-Lara (i) https://orcid.org/0000-0001-6984-4795
L. Sánchez-Menguiano (1) https://orcid.org/0000-00031888-6578

S. F. Sánchez (1) https://orcid.org/0000-0001-6444-9307

C. J. Walcher (1) https://orcid.org/0000-0002-2715-796X

\section{References}

Abazajian, K. N., Adelman-McCarthy, J. K., Agüeros, M. A., et al. 2009, ApJS, 182, 543

Abramson, L. E., Kelson, D. D., Dressler, A., et al. 2014, ApJL, 785, L36

Aguerri, J. A. L., Elias-Rosa, N., Corsini, E. M., \& Muñoz-Tuñón, C. 2005, A\&A, 434, 109

Baldwin, J. A., Phillips, M. M., \& Terlevich, R. 1981, PASP, 93, 5

Barnes, J. E., \& Hernquist, L. E. 1991, ApJL, 370, L65

Bialas, D., Lisker, T., Olczak, C., Spurzem, R., \& Kotulla, R. 2015, A\&A, 576, A103

Book, L. G., \& Benson, A. J. 2010, ApJ, 716, 810

Boselli, A., \& Gavazzi, G. 2006, PASP, 118, 517

Bower, R. G., Benson, A. J., \& Crain, R. A. 2012, MNRAS, 422, 2816

Bruzual, G., \& Charlot, S. 2003, MNRAS, 344, 1000

Cano-Díaz, M., Sánchez, S. F., Zibetti, S., et al. 2016, ApJL, 821, L26

Carles, C., Martel, H., Ellison, S. L., \& Kawata, D. 2016, MNRAS, 463, 1074

Carniani, S., Marconi, A., Maiolino, R., et al. 2016, A\&A, 591, A28

Catalán-Torrecilla, C., Gil de Paz, A., Castillo-Morales, A., et al. 2015, A\&A, 584, A87

Chabrier, G. 2003, PASP, 115, 763

Charlot, S., \& Fall, S. M. 2000, ApJ, 539, 718

Chilingarian, I. V., Cayatte, V., Durret, F., et al. 2008, A\&A, 486, 85

Cid Fernandes, R., Stasińska, G., Mateus, A., \& Vale Asari, N. 2011, MNRAS, 413, 1687

Cid Fernandes, R., Stasińska, G., Schlickmann, M. S., et al. 2010, MNRAS, 403, 1036

Coelho, P., \& Gadotti, D. A. 2011, ApJL, 743, L13

Daddi, E., Dickinson, M., Morrison, G., et al. 2007, ApJ, 670, 156

Dalla Vecchia, C., \& Schaye, J. 2008, MNRAS, 387, 1431

Dalton, G., Trager, S., Abrams, D. C., et al. 2014, Proc. SPIE, 9147, 91470L

de Jong, T., Clegg, P. E., Rowan-Robinson, M., et al. 1984, ApJL, 278, L67

Dekel, A., Birnboim, Y., Engel, G., et al. 2009, Natur, 457, 451

de Souza, R. E., Gadotti, D. A., \& dos Anjos, S. 2004, ApJS, 153, 411

Devereux, N. 1987, ApJ, 323, 91

Dressler, A. 1980, ApJ, 236, 351

Duarte Puertas, S., Vilchez, J. M., Iglesias-Paramo, J., et al. 2017, A\&A, 599, A71

Elbaz, D., Daddi, E., Le Borgne, D., et al. 2007, A\&A, 468, 33

Elbaz, D., Dickinson, M., Hwang, H. S., et al. 2011, A\&A, 533, A119

Ellison, S. L., Nair, P., Patton, D. R., et al. 2011, MNRAS, 416, 2182

Emsellem, E., Cappellari, M., Krajnović, D., et al. 2011, MNRAS, 414, 888

Faber, S. M., \& Jackson, R. E. 1976, ApJ, 204, 668

Falcón-Barroso, J., Lyubenova, M., van de Ven, G., et al. 2017, A\&A, 597, A48

Falcón-Barroso, J., van de Ven, G., Peletier, R. F., et al. 2011, MNRAS, 417, 1787

Fisher, D. B. 2006, ApJL, 642, L17

Fisher, D. B., \& Drory, N. 2008, AJ, 136, 773

Fisher, D. B., \& Drory, N. 2016, Astrophysics and Space Science Library, 418,41

Florido, E., Zurita, A., Pérez, I., et al. 2015, A\&A, 584, A88

Gadotti, D. A. 2009, MNRAS, 393, 1531

Gil de Paz, A., Boissier, S., Madore, B. F., et al. 2007, ApJS, 173, 185

Gil de Paz, A., Carrasco, E., Gallego, J., et al. 2016, Proc. SPIE, 9908, 99081K

Gomes, J. M., Papaderos, P., Kehrig, C., et al. 2016, A\&A, 588, A68

Gómez, P. L., Nichol, R. C., Miller, C. J., et al. 2003, ApJ, 584, 210

González Delgado, R. M., Cid Fernandes, R., Pérez, E., et al. 2016, A\&A, 590, A44

Hao, L., Jogee, S., Barazza, F. D., Marinova, I., \& Shen, J. 2009, in ASP Conf. Ser. 419, Galaxy Evolution: Emerging Insights and Future Challenges, ed. S. Jogee et al. (San Francisco, CA: ASP), 402

Hashimoto, Y., Oemler, A., Jr., Lin, H., \& Tucker, D. L. 1998, ApJ, 499, 589

Ho, L. C., Filippenko, A. V., \& Sargent, W. L. W. 1993, ApJ, 417, 63

Hopkins, P. F., Quataert, E., \& Murray, N. 2012, MNRAS, 421, 3522

Hopkins, P. F., Torrey, P., Faucher-Giguère, C.-A., Quataert, E., \& Murray, N. 2016, MNRAS, 458, 816 
Jarrett, T. H., Chester, T., Cutri, R., et al. 2000, AJ, 119, 2498

Johnston, E. J., Häußler, B., Aragón-Salamanca, A., et al. 2017, MNRAS, 465,2317

Kauffmann, G., Heckman, T. M., Tremonti, C., et al. 2003, MNRAS, 346, 1055

Kawata, D., \& Mulchaey, J. S. 2008, ApJL, 672, L103

Kelz, A., Verheijen, M. A. W., Roth, M. M., et al. 2006, PASP, 118, 129

Kennicutt, R. C., \& Evans, N. J. 2012, ARA\&A, 50, 531

Kewley, L. J., Dopita, M. A., Sutherland, R. S., Heisler, C. A., \& Trevena, J. 2001, ApJ, 556, 121

Kormendy, J., \& Kennicutt, R. C., Jr. 2004, ARA\&A, 42, 603

Koyama, Y., Smail, I., Kurk, J., et al. 2013, MNRAS, 434, 423

Kroupa, P. 2001, MNRAS, 322, 231

Lee, N., Sanders, D. B., Casey, C. M., et al. 2015, ApJ, 801, 80

Leslie, S. K., Kewley, L. J., Sanders, D. B., \& Lee, N. 2016, MNRAS, 455, L82

Madau, P., \& Dickinson, M. 2014, ARA\&A, 52, 415

Magnelli, B., Lutz, D., Saintonge, A., et al. 2014, A\&A, 561, A86

Martig, M., Bournaud, F., Teyssier, R., \& Dekel, A. 2009, ApJ, 707, 250

Martin, D. C., Fanson, J., Schiminovich, D., et al. 2005, ApJL, 619, L1

Meert, A., Vikram, V., \& Bernardi, M. 2015, MNRAS, 446, 3943

Meert, A., Vikram, V., \& Bernardi, M. 2016, MNRAS, 455, 2440

Méndez-Abreu, J., Aguerri, J. A. L., Corsini, E. M., \& Simonneau, E. 2008, A\&A, 478, 353

Méndez-Abreu, J., Debattista, V. P., Corsini, E. M., \& Aguerri, J. A. L. 2014, A\&A, 572, A25

Mendez-Abreu, J., Ruiz-Lara, T., Sanchez-Menguiano, L., et al. 2017, A\&A, 598, A32

Moles, M., Marquez, I., \& Perez, E. 1995, ApJ, 438, 604

Moore, B., Katz, N., Lake, G., Dressler, A., \& Oemler, A. 1996, Natur, 379,613

Moore, B., Lake, G., \& Katz, N. 1998, ApJ, 495, 139

Morselli, L., Renzini, A., Popesso, P., \& Erfanianfar, G. 2016, MNRAS, 462, 2355

Mulchaey, J. S., \& Regan, M. W. 1997, ApJL, 482, L135

Noeske, K. G., Weiner, B. J., Faber, S. M., et al. 2007, ApJL, 660, L43

Oppenheimer, B. D., Davé, R., Kereš, D., et al. 2010, MNRAS, 406, 2325

Page, M. J., Symeonidis, M., Vieira, J. D., et al. 2012, Natur, 485, 213

Peng, Y., Maiolino, R., \& Cochrane, R. 2015, Natur, 521, 192

Renzini, A., \& Peng, Y.-j. 2015, ApJL, 801, L29

Roth, M. M., Kelz, A., Fechner, T., et al. 2005, PASP, 117, 620

Sakamoto, K., Okumura, S. K., Ishizuki, S., \& Scoville, N. Z. 1999, ApJ, 525, 691

Salo, H., Laurikainen, E., Laine, J., et al. 2015, ApJS, 219, 4
Salvatier, J., Wiecki, T. V., \& Fonnesbeck, C. 2016, PeerJ Computer Science, 2, e55

Sánchez, S. F., García-Benito, R., Zibetti, S., et al. 2016, A\&A, 594, A36

Sánchez, S. F., Kennicutt, R. C., Gil de Paz, A., et al. 2012, A\&A, 538, A8

Sánchez Almeida, J., Elmegreen, B. G., Muñoz-Tuñón, C., \& Elmegreen, D. M. 2014, A\&ARv, 22, 71

Sánchez-Blázquez, P., Peletier, R. F., Jiménez-Vicente, J., et al. 2006, MNRAS, 371, 703

Sheth, K., Vogel, S. N., Regan, M. W., Thornley, M. D., \& Teuben, P. J. 2005, ApJ, 632, 217

Shimizu, T. T., Mushotzky, R. F., Meléndez, M., Koss, M., \& Rosario, D. J. 2015, MNRAS, 452, 1841

Silk, J. 2005, MNRAS, 364, 1337

Silk, J. 2013, ApJ, 772, 112

Silk, J., \& Mamon, G. A. 2012, RAA, 12, 917

Singh, R., van de Ven, G., Jahnke, K., et al. 2013, A\&A, 558, A43

Speagle, J. S., Steinhardt, C. L., Capak, P. L., \& Silverman, J. D. 2014, ApJS, 214,15

Stasińska, G., Vale Asari, N., Cid Fernandes, R., et al. 2008, MNRAS, 391, L29

Steinhauser, D., Schindler, S., \& Springel, V. 2016, A\&A, 591, A51

Stringer, M. J., Bower, R. G., Cole, S., Frenk, C. S., \& Theuns, T. 2012, MNRAS, 423, 1596

Tabor, M., Merrifield, M., Aragón-Salamanca, A., et al. 2017, MNRAS, 466, 2024

Taylor, E. N., Hopkins, A. M., Baldry, I. K., et al. 2011, MNRAS, 418, 1587

Terashima, Y., Ho, L. C., \& Ptak, A. F. 2000, ApJ, 539, 161

Tonnesen, S., \& Cen, R. 2014, ApJ, 788, 133

Tran, H. D. 2001, ApJL, 554, L19

Tran, H. D., Lyke, J. E., \& Mader, J. A. 2011, ApJL, 726, L21

Vazdekis, A., Sánchez-Blázquez, P., Falcón-Barroso, J., et al. 2010, MNRAS, 404, 1639

Walcher, C. J., Wisotzki, L., Bekeraité, S., et al. 2014, A\&A, 569, A1

Wang, J., Kauffmann, G., Overzier, R., et al. 2012, MNRAS, 423, 3486

Weinberger, R., Springel, V., Hernquist, L., et al. 2017, MNRAS, 465, 3291

Weinzirl, T., Jogee, S., Khochfar, S., Burkert, A., \& Kormendy, J. 2009, ApJ, 696, 411

Whitaker, K. E., Franx, M., Bezanson, R., et al. 2015, ApJL, 811, L12

Whitaker, K. E., Franx, M., Leja, J., et al. 2014, ApJ, 795, 104

Whitaker, K. E., van Dokkum, P. G., Brammer, G., \& Franx, M. 2012, ApJL, 754, L29

Wuyts, S., Förster Schreiber, N. M., van der Wel, A., et al. 2011, ApJ, 742, 96 